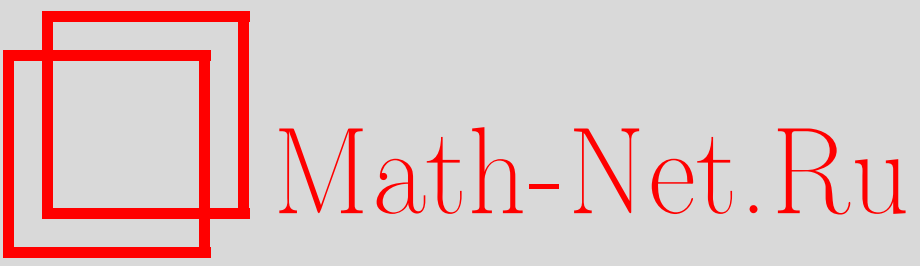

Д. А. Лейтес, А. Н. Сергеев, Ортогональные многочлены дискретной переменной и алгебры Ли матриц комплексного порядка, ТМФ, 2000, том 123, номер 2, 205-236

DOI: https://doi.org/10.4213/tmf599

Использование Общероссийского математического портала Math-Net.Ru подразумевает, что вы прочитали и согласны с пользовательским соглашением

http: //www . mathnet.ru/rus/agreement

Параметры загрузки:

IP : 34.227 .88 .159

26 апреля 2023 г., 12:03:30 


\section{ОРТОГОНАЛЬНЫЕ МНОГОЧЛЕНЫ ДИСКРЕТНОЙ ПЕРЕМЕННОЙ И АЛГЕБРЫ ЛИ МАТРИЦ КОМПЛЕКСНОГО ПОРЯДКА}

Предлагается единая интерпретация классических непрерывных многочленов Чебышева и ортогональных многочленов Хана дискретной переменной в терминах фейгиновской алгебры Ли $\mathfrak{g l}(\lambda)$ при $\lambda \in \mathbb{C}$. $q$-Многочлены Чебышева и Хана можно интерпретировать подобным же образом и ввести ортогональные многочлены, соответствующие супералгебрам Ли. Кроме того, описаны квазиконечные модули над алгеброй $\mathfrak{g l}(\lambda)$, вещественная форма этой алгебры и условия унитарности квазиконечных модулей. Вводится также аналог тензоров над $\mathfrak{g l}(\lambda)$.

\section{1. ВВЕДЕНИЕ}

Эта работа представляет собой доклад памяти Миши Савельева. Работа состоит из трех частей: описание ортогональных многочленов (разделы $1,8,9$ ), описание производяшей функции следа и ее аналогов (раздел 2) и описание квазиконечных модулей над алгеброй Ли $\mathfrak{g l}(\lambda)$ при $\lambda \in \mathbb{C}$ (разделы $3-7)$.

Уравнение вида

$$
\sigma(x) y^{\prime \prime}+\tau(x) y^{\prime}+\lambda y=0,
$$

где $\sigma(x)$ - многочлен степени $2, \tau(x)$ - многочлен степени 1 , а $\lambda$ - константа, называется уравнением гипергеометрического типа, а его решения называются функциями гипергеометрического типа. Умножая уравнение (1.1) на подходящую функцию $\rho(x)$, его можно привести к самосопряженному виду

$$
\left(\sigma(x) \rho(x) y^{\prime}\right)^{\prime}+\lambda \rho(x) y=0, \quad \text { где }(\sigma(x) \rho(x))^{\prime}=\tau(x) \rho(x) .
$$

Пусть $y_{m}$ и $y_{n}$ - решения уравнения (1.2), которым соответствуют различные собственные значения $\lambda_{m}$ и $\lambda_{n}$. Если для некоторых, не обязательно конечных, чисел $a$ и $b$ функция $\rho(x)$ удовлетворяет условиям

$$
\left.\sigma(x) \rho(x) x^{k}\right|_{x=a, b}=0 \quad \text { при } \quad k=0,1, \ldots,
$$

\footnotetext{
*Department of Mathematics, University of Stockholm, Stockholm, Sweden. E-mail: mleites@matematik.su.se

${ }^{\dagger}$ Балаковский университет техники, технологии и контроля, Балаково, Саратовская обл., Россия
} 
то

$$
\int_{a}^{b} y_{m}(x) y_{n}(x) \rho(x) d x=0 .
$$

(Очевидно, если $a$ и $b$ конечны, достаточно потребовать, чтобы выполнялось условие $\left.\left.\sigma(x) \rho(x)\right|_{x=a, b}=0.\right)$

Примером функции гипергеометрического типа являются многочлены Якоби $P_{n}^{(\alpha, \beta)}(x)$, определенные при $\alpha, \beta>-1$ как полиномиальные решения уравнения (1.2) для $\sigma(x)=1-x^{2}, \rho=(1-x)^{\alpha}(1+x)^{\beta},(a, b)=(-1,1)$ и $\tau=\beta-\alpha-(\alpha+\beta+2) x$. При $\alpha=\beta=0$ многочлены Якоби называются многочленами Чебышева.

Разностное уравнение, аппроксимируюшее уравнение (1.1) на однородной решетке, очевидно, имеет вид

$$
\begin{aligned}
\sigma(x) \frac{1}{h} & {\left[\frac{y(x+h)-y(x)}{h}-\frac{y(x)-y(x-h)}{h}\right]+} \\
& +\frac{\tau(x)}{2}\left[\frac{y(x+h)-y(x)}{h}+\frac{y(x)-y(x-h)}{h}\right]+\lambda y=0 .
\end{aligned}
$$

Положим $h=1$ и $\Delta f(x)=f(x+1)-f(x), \nabla f(x)=f(x)-f(x-1)$. Тогда разностное уравнение (1.4) принимает вид

$$
\Delta(\sigma(x) \rho(x) \nabla y)+\lambda \rho(x) y=0, \quad \text { где } \quad \Delta(\sigma(x) \rho(x))=\tau(x) \rho(x) .
$$

Соотношения ортогональности

$$
\sum_{x_{i}=a}^{b-1} y_{m}\left(x_{i}\right) y_{n}\left(x_{i}\right) \rho\left(x_{i}\right)=0
$$

выполняются, если функция $\rho(x)$ удовлетворяет условиям

$$
\left.\sigma(x) \rho(x) x^{k}\right|_{x=a, b}=0 \quad \text { при } \quad k=0,1, \ldots
$$

Многочлен Хана $h_{n}^{(\alpha, \beta)}(x, N)$ представляет собой полиномиальное решение уравнения (1.5), определенное при $\alpha, \beta>-1$ для $\sigma(x)=x(N+\alpha-x)$,

$$
\rho=\frac{\Gamma(N+\alpha-x) \Gamma(\beta+1+x)}{\Gamma(x+1) \Gamma(N x)},
$$

$(a, b)=(0, N)$ и $\tau=(\beta+1)(N-1)-(\alpha+\beta+2) x$. Как было отмечено в работе [1], многочлены Хана были в действительности известны и Чебышеву и изучались им; Хан переоткрыл их и их $q$-аналоги.

Многочлен Чебъшева $t_{n}(x, N)$ представляет собой полиномиальное решение уравнения (1.5), определенное при $\alpha=\beta=0, \sigma(x)=x(N-x), \rho=1,(a, b)=(0, N)$ и $\tau=N-1-2 x$.

Обычный способ получения $q$-аналогов этих многочленов заключается в рассмотрении неоднородных разбиений отрезка $[a, b]$ [2]. Другой способ состоит в применении 
нашего подхода к алгебре $U_{q}(\mathfrak{s l}(2))$ вместо алгебры $U(\mathfrak{s l}(2))$, которая рассматривается в данной работе.

До настоящего времени предполагалось, что стандартные внутренние произведения (1.3) и (1.6) положительно определены. Хотя $N$, число узлов при однородном разбиении отрезка $(a, b)$, является целым, в выражениях для многочленов $h_{n}^{(\alpha, \beta)}(x, N)$ и $t_{n}(x, N)$ его можно заменить любым комплексным числом, поскольку эти многочлены зависят от $N$ аналитически. Было замечено [2], что для чисто мнимых $N$ имеется мера, приводящая к положительно-определенному произведению типа (1.3). Были открыты также многочисленные тождества, которым удовлетворяют ортогональные многочлены; полнота списка этих тождеств никогда не обсуждалась.

Дадим резюме полученных в данной работе результатов. Мы обнаружили, что определение непрерывных многочленов Чебышева и ортогональных многочленов Хана дискретной переменной (и их вариантов для неоднородных решеток, или в современной терминологии $q$-аналогов), а также все тождества, которым они удовлетворяют, следуют из свойств элементов Казимира алгебры Ли $\mathfrak{g l}(\lambda)$ матриц комплексного порядка (и ее $q$-аналога). Отправной точкой нашего подхода была попытка вычислить в явном виде значение квадратичного оператора Казимира на квазиконечных $\mathfrak{g l}(\lambda)$-модулях, являющееся источником ортогональных соотношений и различных тож деств, которым удовлетворяют вышеупомянутые многочлены. Более подробное описание квазиконечных модулей уровня 1 можно найти в работе [3].

Пусть число $\lambda=n>0$ целое. Инвариантный функционал (след) на алгебре $\mathfrak{G}=$ $\mathfrak{g l}(n)$ порождает невырожденную билинейную форму $(A, B)=\operatorname{tr} A B$. Однородные компоненты $\mathfrak{G}_{i}$, где $\mathfrak{G}_{i}-$ пространство матриц с носителем на $i$-й наддиагонали (при $\left.i>0\right)$ или поддиагонали (при $i<0$ ), ортогональны относительно этой формы, и форма являетя невырожденной на $\mathfrak{G}_{-i} \oplus \mathfrak{G}_{i}$ и на $\mathfrak{G}_{0}$. Приведение билинейной формы к каноническому виду на таких пространствах дает классические многочлены дискретной переменной Хана и Чебышева соответственно. Выражая элементы Казимира (генераторы центра алгебры $U(\mathfrak{G}))$ в терминах таких многочленов в стандартном базисе, получаем все тождества, которым эти многочлены удовлетворяют. Более точно, мы выражаем элементы как пространства $\mathfrak{G}_{0}$, так и пространства $\mathfrak{G}_{-i} \oplus \mathfrak{G}_{i}$ в терминах многочленов от одной переменной $H$. Детали приводятся в основном тексте статьи, здесь же мы только очертим идею.

Рассмотрим базис

$$
Y=\left(\begin{array}{ll}
0 & 0 \\
1 & 0
\end{array}\right), \quad H=\left(\begin{array}{cc}
1 & 0 \\
0 & -1
\end{array}\right), \quad X=\left(\begin{array}{ll}
0 & 1 \\
0 & 0
\end{array}\right)
$$

в алгебре $\mathfrak{s l}(2)$ с коммутационными соотношениями

$$
[X, Y]=H, \quad[H, Y]=-2 Y, \quad[H, X]=2 X .
$$

Рассмотрим главное вложение алебры $\mathfrak{s l}(2)$ в алгебру $\mathfrak{G}=\mathfrak{g l}(n)$ (это вложение соответствует $n$-мерному неприводимому $\mathfrak{s l}(2)$-модулю):

$$
Y \mapsto \sum_{i=1}^{n-1} E_{i+1, i}, \quad H \mapsto \sum_{i=1}^{n}(n-2 i+1) E_{i i}, \quad X \mapsto \sum_{i=1}^{n-1} i(n-i) E_{i, i+1} .
$$


Тогда $\mathfrak{G}$ имеет вид

$$
\mathfrak{G}=\bigoplus_{|i| \leqslant n-1} \mathfrak{G}_{i}
$$

где $\mathfrak{G}_{i}=\left\{X^{i} \mathfrak{G}_{0}\right\}$ и $\mathfrak{G}_{-i}=\left\{\mathfrak{G}_{0} Y^{i}\right\}$ при $i>0$, а $\mathfrak{G}_{0}=\mathbb{C}[H] /\left(P_{n}(H)\right)$ - подалгебра Картана; здесь

$$
P_{n}(H)=\prod_{1 \leqslant i \leqslant n}(H-n+2 i-1) .
$$

Применяя данный подход к алгебре $\mathfrak{g l}(\lambda), \lambda \in \mathbb{C}$, мы проясняем известные результаты относительно так называемых непрерывных вариантов классических многочленов дискретной переменной Хана и Чебышева (см. работы $[2,4])$, относящиеся к чисто мнимым значениям $N=\lambda$, и получаем некоторые новые результаты. Таким образом, мы начинаем с объяснения того, что такое алгебра Ли $\mathfrak{g l}(\lambda)$, введенная в работе [5].

Квадратичный оператор Казимира алгебры $\mathfrak{s l}(2)$

$$
\Omega=2 Y X+\frac{1}{2} H^{2}+H
$$

лежит в центре алгебры $U(\mathfrak{s l}(2))$. Пусть $I_{\lambda}-$ двусторонний идеал в ассоциативной алгебре $U(\mathfrak{s l}(2))$, порожденной элементом $\Omega-\left(\lambda^{2}-1\right) / 2$. Оказывается, что ассоциативная алгебра $\widetilde{\mathfrak{A}}_{\lambda}=U(\mathfrak{s l}(2)) / I_{\lambda}$ проста при $\lambda \notin \mathbb{Z} \backslash\{0\}$, в остальных случаях $\widetilde{\mathfrak{A}}_{\lambda}$ содержит идеал такой, что фактор по нему изоморфен матричной алгебре $\operatorname{Mat}(|\lambda|)$. Положим $[6]$

$$
\mathfrak{A}_{\lambda}= \begin{cases}\tilde{\mathfrak{A}}_{\lambda} & \text { при } \lambda \notin \mathbb{Z} \backslash\{0\}, \\ \operatorname{Mat}(|\lambda|) & \text { в других случаях. }\end{cases}
$$

Ассоциативная алгебра с единицей $\mathfrak{A}_{\lambda}$ порождается элементами $X, Y$ и $H$, удовлетворяюшими соотношениям (1.7), а также соотношению

$$
X Y=\frac{1}{4}\left(\lambda^{2}-(H-1)^{2}\right)
$$

и еше одному соотношению при целых $\lambda$

$$
X^{|\lambda|}=0 \quad \text { при } \quad \lambda \in \mathbb{Z} \backslash\{0\} .
$$

В дальнейшем полагаем $\mathfrak{g l}(\lambda):=L\left(\mathfrak{A}_{\lambda}\right)$, что дает алгебру Ли, получаемую из ассоциативной алгебры $\mathfrak{A}_{\lambda}$, при замене произведения, обозначаемого точкой, на произведение, обозначаемое скобками.

Если в соотношении (1.9) заменить $U(\mathfrak{s l}(2))$ на ее $q$-деформацию $U_{q}(\mathfrak{s l}(2))$, мы получим $q$-варианты классических многочленов, тождеств и т.д. Соответствующие подробности будут опубликованы отдельно.

Классики рассматривали ортогональные многочлены относительно знакоопределенного скалярного произведения, задаваемого мерой или соответствующей суммой для разностных уравнений. В традиционных подходах существование такой меры для комплексных значений некоторых параметров неочевидно $[2,4,7]$. В нашем подходе явно видно, что мера сушествует при любых $\lambda \in \mathbb{Z} \backslash\{0\}$ и при чисто мнимых $\lambda$ (см. ниже 
формулу (9.2)). Видно, что формула (9.2) является знакоопределенной при вешественных $\lambda$ таких, что $0<|\lambda|<1$.

Поскольку в точке общего положения скалярное произведение не знакоопределено, естественно также рассматривать и такие скалярные произведения. Более того, если заменить в приведенной выше конструкции алгебру $\mathfrak{s l}(2)$ супералгеброй Ли $\mathfrak{g}$, мы никогда не получим знакоопределеное скалярное произведение на супералгебре Картана. Незнакоопределенные (но невырожденные и симметричные) скалярные произведения рассматривались в литературе. Соответствуюшие ортогональные многочлены удовлетворяют дифференциальным уравнениям порядка, большего чем 2 [8-10] (мы благодарны Т. Я. Азизову, указавшему нам на эти работы). Подобные уравнения, вероятно, описывают что-то, имеюшее отношение к действительности. Васильев в работе [11] показал возможную область применимости этих уравнений. Тем не менее физики предпочитают уравнения второго порядка, поскольку они описывают большинство реальных процессов.

В известных нам попытках обобшить классические непрерывные многочлены дискретной переменной на случай нескольких переменных в действительности рассматривается представление прямой суммы нескольких копий алгебры $\mathfrak{A}_{\lambda}$ (см. (1.9)) в тензорном произведении модулей Верма над $\mathfrak{s l}(2)$. Это ведет к суммам произведений многочленов от одной переменной, т.е. к несколько более сложным многочленам, но все же многочленам от одной переменной.

Наш подход приводит к многочленам гораздо более сложной структуры, а именно мы предлагаем в описанном вьше подходе заменить алгебру $\mathfrak{s l}(2)$ на любую алгебру Ли или супералгебру Ли g. Это удается сделать при условии, что $\mathfrak{g}$ обладает, во-первых, модулями Верма с большим, но конечным числом генераторов и, во-вторых, несколькими конечномерными представлениями. По-видимому, теория является наиболее богатой, если алгебра $\mathfrak{g}$ простая или близкая к простой (нетривиальное центральное расширение, производная алгебра и т.д.). Такие обобшения алгебр $\mathfrak{g l}(\lambda)$ появляются в моделях спина большего 2 и в модели Калоджеро-Сазерленда [11]. Результаты, полученные таким способом, будут рассмотрены в отдельной работе. В заключение отметим, что недавно один из авторов (А. С.) нашел, как охватить все классические многочлены дискретной переменной, а не только многочлены Чебышева и Хана, способом, несколько более общим, чем описанньй в данной работе.

\section{2. ФОРМУЛА СЛЕДА}

Лемма 2.1. Пусть $A$ - ассочиативная алгебра, порождаемая алгеброй Ли $\mathfrak{g}$, рассматриваемой как подпространство, т.е. $A$ - фактор алгебры $U(\mathfrak{g})$. Тогда $[A, A]=[\mathfrak{g}, A]$.

ДокаЗАТЕЛЬСтво. Достаточно показать, что $\left[x_{1} \ldots x_{n}, a\right] \in[\mathfrak{g}, A]$ для любых $a \in A$ и $x_{1}, \ldots, x_{n} \in \mathfrak{g}$. Проведем индукцию по $n$. При $n=1$ утверждение очевидно. Пусть $n>1$. Воспользуемся тождеством $[a b, c]=[a, b c]+[b, c a]$, имеюшим место в любой ассоциативной алгебре (см. работу [12]). Тогда

$$
\left[x_{1}\left(x_{2} \ldots x_{n}\right), a\right]=\left[x_{1}, x_{2} \ldots x_{n} a\right] \pm\left[x_{2} \ldots x_{n}, a x_{1}\right] .
$$

2 Теоретическая и математическая физика, т. 123, № 2, 2000 г. 
По предположению индукции второе слагаемое в правой части лежит в $[\mathfrak{g}, A]$. Лемма доказана.

СЛЕДСТВИЕ 2.1. Пусть $\mathfrak{g}$ - простая алгебра Ли или алгебра osp $(1 \mid 2 n)$. Тогда $U(\mathfrak{g})=Z(U(\mathfrak{g})) \oplus[U(\mathfrak{g}), U(\mathfrak{g})]$.

ДокАЗАТЕЛЬСТво. Пусть

$$
U(\mathfrak{g})=Z(U(\mathfrak{g})) \oplus\left(\bigoplus_{\lambda \neq 0} n_{\lambda} L^{\lambda}\right)
$$

- разложение на неприводимые $\mathfrak{g}$-модули относительно присоединенного представления. Поскольку модули $L^{\lambda}$ неприводимы,

$$
[\mathfrak{g}, U(\mathfrak{g})]=\left(\bigoplus_{\lambda \neq 0} n_{\lambda} L^{\lambda}\right)
$$

(этот аргумент не работает для супералгебр Ли, отличных от osp $(1 \mid 2 n)$, из-за отсутствия полной приводимости), что и требовалось доказать.

В дальнейшем $\rho$ равно полусумме положительных корней.

СлЕДСТВИЕ 2.2. Пусть $\mathfrak{h}$ - подалгебра Картана в алгебре $\mathfrak{g}$, которая является или простой алгеброй, или конечномерной алгеброй Ли; пусть $\lambda \in \mathfrak{h}^{*}$ u $M^{\lambda-\rho}-$ модуль Верма со старшим весом $\lambda($ не $\lambda-\rho)$; пусть $J_{\lambda}-($ левый) идеал алгебры $U(\mathfrak{g})$, равньй ядру представления $U(\mathfrak{g})$ в $M^{\lambda-\rho}$. Положим $\mathfrak{A}_{\lambda}=U(\mathfrak{g}) / J_{\lambda}$. Тогда $\mathfrak{A}_{\lambda}=\mathbb{C} \oplus\left[\mathfrak{A}_{\lambda}, \mathfrak{A}_{\lambda}\right]$.

ДокаЗАТЕльство. Согласно лемме $2.1,\left[\mathfrak{A}_{\lambda}, \mathfrak{A}_{\lambda}\right]=\left[\mathfrak{g}, \mathfrak{A}_{\lambda}\right] ;$ следовательно, как в следствии $2.1, \mathfrak{A}_{\lambda}=Z\left(\mathfrak{A}_{\lambda}\right) \oplus\left[\mathfrak{A}_{\lambda}, \mathfrak{A}_{\lambda}\right]$. Однако $Z\left(\mathfrak{A}_{\lambda}\right)$ - гомоморфный образ $Z(U(\mathfrak{g}))$; следовательно, он равен $\mathbb{C}$, что и требовалось доказать.

Если $\mathfrak{g}$ - простая алгебра Ли, а $\lambda$ - старший вес конечномерного модуля, то $P(\lambda)=$ $\operatorname{dim} L^{\lambda}-$ многочлен от $\lambda=\left(\lambda_{1}, \ldots, \lambda_{n}\right)$. Рассмотрим $P(\lambda)$ для любых $\lambda \in \mathfrak{h}^{*}$ таких, что

$$
\prod_{i \neq j}\left(\lambda_{i}-\lambda_{j}\right) \neq 0 .
$$

Согласно следствию 2.2 имеется только один функционал $\operatorname{tr}$ на $\mathfrak{A}_{\lambda}$ такой, что $\operatorname{tr}(a b)=$ $\operatorname{tr}(b a)$ и $\operatorname{tr}(1)=P(\lambda)$.

Лемма 2.2. Пусть $u \in U(\mathfrak{g})$, a $\tilde{u} \in \mathfrak{A}_{\lambda}-$ образ этого әлемента. Тогда $\operatorname{tr}(\tilde{u})-$ также многочлен от $\lambda$.

ДокАЗАтЕЛЬСтво. Пусть \#: $Z(U(\mathfrak{g})) \oplus[U(\mathfrak{g}), U(\mathfrak{g})] \rightarrow Z(U(\mathfrak{g}))$ - естественная проекция. По определению \# имеем

$$
\operatorname{tr}(\tilde{u})=\chi_{\lambda}\left(u^{\#}\right) P(\lambda)=\varphi\left(u^{\#}\right)(\lambda) P(\lambda),
$$

где $\varphi: Z(U(\mathfrak{g})) \rightarrow \mathbb{C}[\mathfrak{h}]$ - гомоморфизм Хариш-Чандры [13]. Лемма доказана. 
ТеОрема 2.1. Пусть $\mathfrak{g}$ - простая алгебра Ли, а $\mathfrak{h}-$ ее подалгебра Картана. Отождествим пространство $(\mathbb{C}[\mathfrak{h}])^{*}$ с алгеброй формальных степенных рядов от переменных $t=\left(t_{1}, \ldots, t_{n}\right)$ следующим образом:

$$
f \mapsto \sum_{\nu=\left(\nu_{1}, \ldots, \nu_{n}\right)} f\left(\frac{h_{1}^{\nu_{1}}}{\nu_{1} !} \ldots \frac{h_{n}^{\nu_{n}}}{\nu_{n} !}\right) t_{1}^{\nu_{1}} \ldots t_{n}^{\nu_{n}}
$$

где $h_{1}, \ldots, h_{n}-$ базис в $\mathfrak{h}$, а $\nu_{i} \in \mathbb{Z}_{+}$для любьх $i$. Пусть $e^{\lambda}(t)=e^{\lambda_{1} t_{1}+\cdots+\lambda_{n} t_{n}} u$ все $\lambda_{i}$ подразумеваются различными. Тогда функиионал $\operatorname{tr}$ соответствует ряду

$$
\psi(\lambda, t)=\frac{\bigoplus_{w \in W} \varepsilon(w) e^{w(\lambda)}}{\bigoplus_{w \in W} \varepsilon(w) e^{w(\rho)}}(t) .
$$

ДокАЗАТЕЛЬСтво. Согласно лемме 2.2

$$
\operatorname{tr}\left(\frac{h_{1}^{\nu_{1}}}{\nu_{1} !} \ldots \frac{h_{n}^{\nu_{n}}}{\nu_{n} !}\right)
$$

- многочлен от $\lambda$. Если $\lambda \in P_{++}$(где $P_{++}-$множество старших весов конечномерных модулей), то ряд

$$
\hat{\psi}(\lambda, t)=\sum_{\nu=\left(\nu_{1}, \ldots, \nu_{n}\right)} \operatorname{tr}\left(\frac{h_{1}^{\nu_{1}}}{\nu_{1} !} \ldots \frac{h_{n}^{\nu_{n}}}{\nu_{n} !}\right) t_{1}^{\nu_{1}} \ldots t_{n}^{\nu_{n}}
$$

согласуется с (2.3) вследствие формулы Вейля для характеров. Однако $\psi(\lambda, t)=$ $\sum_{\nu} P_{\nu} t^{\nu}$, где $P_{\nu}-$ некоторые многочлены. Действительно, поскольку

$$
P_{\nu}(\lambda)=\operatorname{tr}\left(\frac{h_{1}^{\nu_{1}}}{\nu_{1} !} \ldots \frac{h_{n}^{\nu_{n}}}{\nu_{n} !}\right) \quad \text { при } \quad \lambda \in P_{++},
$$

это справедливо при любых $\lambda \in \mathfrak{h}^{*}$. Следовательно, $\hat{\psi}(\lambda, t)=\psi(\lambda, t)$. Теорема доказана.

\section{3. СТАРШИЕ ВЕСА КВАЗИКОНЕЧНЫХ МОДУЛЕЙ НАД АЛГЕБРОЙ $\mathfrak{g l}(\lambda)$}

Пусть $L^{i}$ - неприводимый $\mathfrak{s l}(2)$-модуль со старшим весом $i$. Алгебра Ли $\mathfrak{g l}(\lambda)$, рассматриваемая как $\mathfrak{s l}(2)$-модуль относительно присоединенного действия $\mathfrak{s l}(2)$, имеет вид

$$
\mathfrak{g l}(\lambda)= \begin{cases}L^{0} \oplus L^{2} \oplus \cdots \oplus L^{2|\lambda|-2} & \text { при } \lambda \in \mathbb{Z} \backslash\{0\}, \\ L^{0} \oplus L^{2} \oplus \cdots & \text { в других случаях. }\end{cases}
$$

АлгебраЛи $\mathfrak{g l}(\lambda)$ обладает $\mathbb{Z}$-градуировкой

$$
\mathfrak{g l}(\lambda)=\bigoplus_{i \in \mathbb{Z}} \mathfrak{g l}(\lambda)_{i}
$$

где

$$
\mathfrak{g l}(\lambda)_{i}=\{z \in \mathfrak{g l}(\lambda):[H, z]=2 i z\}
$$


и возрастающей фильтрацией

$$
\mathfrak{g l}(\lambda)_{(i)}=\bigoplus_{k \leqslant i} \mathfrak{g l}(\lambda)_{k}
$$

Таким образом, мы можем говорить о треугольном разложении $\mathfrak{g}=\mathfrak{g}_{-} \oplus \mathfrak{g}_{0} \oplus \mathfrak{g}_{+}$ алгебры $\mathfrak{g}=\mathfrak{g l}(\lambda)$, где

$$
\mathfrak{g}_{ \pm}=\bigoplus_{i \geqslant 0} \mathfrak{g l}(\lambda)_{ \pm i}
$$

а также о параболических подалгебрах, стариих весах и т.д. [14].

Следуя работе [14], назовем $\mathfrak{g}$-модуль $V$ квазиконечным , если $V=\bigoplus_{j \in \mathbb{Z}} V_{j}$ и $\operatorname{dim} V_{j}<\infty$ для любых $j$. Мы рассматриваем только градуированные $\mathfrak{g}$-модули, т.е. такие, что $\mathfrak{g}_{i} V_{j} \subset V_{i+j}$.

Хорошо известный факт, что $\mathfrak{g l}(n)$ имеет нетривиальное одномерное представление $(A \mapsto \operatorname{tr} A)$, имеет свой аналог и для $\mathfrak{g l}(\lambda)$.

Подалгебра $\mathfrak{p} \subset \mathfrak{g}$ называется параболической, если она содержит $\mathfrak{g}_{0} \oplus \mathfrak{g}_{+}$как собственную подалгебру. Например, для любого $P \in \mathbb{C}[H]$ мы полагаем $\mathfrak{p}_{-1}(P)=P \mathbb{C}[H] Y$ и считаем, что $\mathfrak{p}(P)$ порождается $\mathfrak{p}_{-1}(P)$ и $\mathfrak{g}_{0} \bigoplus \mathfrak{g}_{+}$. Это дает минимальную параболическую подалгебру, соответствуюшую $P$.

ЛЕмма 3.1. Минимальная параболическая подалгебра $\mathfrak{p}(P)$ имеет вид

$$
\mathfrak{p}(P)=\bigoplus_{k \in \mathbb{Z}} \mathfrak{p}(P)_{k},
$$

где $\mathfrak{p}(P)_{k}=\mathfrak{g}_{k}$ при $k \geqslant 0 u \mathfrak{p}(P)_{-k}=I_{k} \cdot Y^{k}$, а $I_{k}-$ идеал в $\mathbb{C}[H]$, порожсденньй $P(H) P(H+2) \ldots P(H+2 k-2)$.

ДокаЗАТЕЛЬСтво. См. [14] (mutatis mutandis).

Пусть $\lambda \in \mathfrak{g}_{0}^{*}, \mathfrak{g}_{+} v_{\lambda}=0$ и $M^{\lambda}=\operatorname{ind}_{\mathfrak{g}_{0} \oplus \mathfrak{g}_{+}}^{\mathfrak{g}}\left(\mathbb{C} v_{\lambda}\right)$ - соответствующий модуль Верма; пусть $L^{\lambda}$ - неприводимый $\mathfrak{g}$-модуль со старшим весом $\lambda$.

Лемма 3.2 [14]. Следующие условия әквивалентны:

I. $M^{\lambda}$ содержит вакуумный вектор, который лежст в $M_{(-1)}^{\lambda}-$ пространстве фильтрации -1.

II. Модуль $L^{\lambda}$ является квазиконечным.

III. $L^{\lambda}$ есть фактор обобщенного модуля Верма $M^{\lambda, P}=\operatorname{ind}_{\mathfrak{p}(P)}^{\mathfrak{g}}\left(\mathbb{C} v_{\lambda}\right)$ для некоmорого $P \in \mathbb{C}[H]$.

Шойхет называет $\operatorname{deg} P$ уровнем модуля $M^{\lambda, P}$. В работе [3] он описывает модули уровня 1 более детально, чем мы это делаем здесь.

Мы уже установили, что $\mathfrak{g}_{0}^{*} \simeq(\mathbb{C}[H])^{*} \simeq \mathbb{C}[[t]]$, где изоморфизм алгебр имеет вид

$$
F:(\mathbb{C}[H])^{*} \rightarrow \mathbb{C}[[t]], \quad \theta \mapsto F_{\theta}(t)=\sum_{k=0}^{\infty} \frac{\theta\left(H^{k}\right)}{k !} t^{k}
$$

Следуюшая теорема описывает набор формальных степенных рядов, соответствующих старшим весам квазиконечных модулей. Напомним, что квазимногочлен представляет собой выражение вида $\sum R_{i}(t) e^{\alpha_{i} t}$, где $R_{i}(t)$ - многочлены. 
ТЕОРема 3.1. Формальный степенной ряд, соответствующий стариему весу любого квазиконечного модуля над $\mathfrak{g l}(\lambda)$, имеет вид $R(t) /\left(1-e^{-2 t}\right)$, где $R(t)$ квазимногочлен такой, что $R(0)=0$.

ДокАЗАТЕЛЬСтво. Во-первых, заметим, что легко доказываются следующие утверждения:

а) если $\theta \in(\mathbb{C}[H])^{*}$, a

$$
F_{\theta}(t)=\sum_{k=0}^{\infty} \frac{\theta\left(H^{k}\right)}{k !} t^{k}
$$

- соответствуюший ряд, то

$$
\sum_{k=0}^{\infty} \frac{\theta\left((H+a)^{k}\right)}{k !} t^{k}=e^{a t} F_{\theta}(t)
$$

б) для любого $R(H) \in \mathbb{C}[H]$ имеем

$$
\begin{aligned}
\sum_{k=0}^{\infty} \frac{\theta\left(R(H) H^{k}\right)}{k !} t^{k} & =R\left(\frac{d}{d t}\right) F_{\theta}(t) \\
\sum_{k=0}^{\infty} \frac{\theta\left(R(H+a)(H+a)^{k}\right)}{k !} t^{k} & =R\left(\frac{d}{d t}\right)\left(e^{a t} F_{\theta}(t)\right) .
\end{aligned}
$$

Если $\theta$ - старший вес квазиконечного модуля над $\mathfrak{g l}(\lambda)$, то по лемме 3.2 существует многочлен $P(H) \in \mathbb{C}[H]$ такой, что $\theta$ продолжается на одномерное представление минимальной параболической подалгебры, соответствуюшей $P$. Нетрудно проверить, что $[\mathfrak{p}, \mathfrak{p}] \cap \mathfrak{g}_{0}=\left[\mathfrak{g}_{1}, \mathfrak{p}_{-1}\right]$. Следовательно, $\theta\left(\left[\mathfrak{g}_{1}, \mathfrak{p}_{-1}\right]\right)=0$.

Обозначим

$$
T(H)=X Y=\frac{1}{4}\left(\lambda^{2}-(H+1)^{2}\right) .
$$

Тогда условие $\theta\left(\left[\mathfrak{g}_{1}, \mathfrak{p}_{-1}\right]\right)=0$ можно выразить как $\theta\left(\left[X, P(H) H^{k} Y\right]\right)=0$ или как

$$
\theta\left(T(H-2) P(H-2)(H-2)^{k}-P(H) H^{k} T(H)\right)=0 .
$$

Следовательно,

$$
\begin{aligned}
& \sum_{k=0}^{\infty} \frac{\theta\left(T(H-2) P(H-2)(H-2)^{k}\right)}{k !} t^{k}-\sum_{k=0}^{\infty} \frac{\theta\left(T(H) P(H)(H)^{k}\right)}{k !} t^{k}= \\
& \quad=T\left(\frac{d}{d t}\right) P\left(\frac{d}{d t}\right)\left(e^{-2 t} F_{\theta}(t)\right)-T\left(\frac{d}{d t}\right) P\left(\frac{d}{d t}\right)\left(F_{\theta}(t)\right)= \\
& =T\left(\frac{d}{d t}\right) P\left(\frac{d}{d t}\right)\left(\left(e^{-2 t}-1\right) F_{\theta}(t)\right)=0 .
\end{aligned}
$$

Таким образом, функция $\left(e^{-2 t}-1\right) F_{\theta}(t)$ является решением обыкновенного дифференциального уравнения с постоянными коэффициентами и, следовательно, многочленом. Очевидно, что $R(0)=0$. 
Обратно, если $R(t)$ представляет собой квазимногочлен, $R(0)=0$ и $P(d / d t) R(t)=0$, мы имеем

$$
T\left(\frac{d}{d t}\right) P\left(\frac{d}{d t}\right) R(t)=0
$$

и условие $\theta\left(\left[\mathfrak{g}_{1}, \mathfrak{p}_{-1}\right]\right)=0$ для $\mathfrak{p}_{-1}=P(H) \mathfrak{g}_{0} Y$ удовлетворяется, если мы положим

$$
F_{\theta}(t)=\frac{R(t)}{e^{-2 t}-1}
$$

(что корректно определено, поскольку $R(0)=0$ ). Следовательно, $\theta$ является старшим весом квазиконечного модуля. Теорема доказана.

Рассмотрим след на $\mathfrak{g l}(0)$. Если $P=1$, то параболическая подалгебра в $\mathfrak{g}=\mathfrak{g l}(0)$ совпадает с полной алгеброй и поэтому $[\mathfrak{g}, \mathfrak{g}] \cap \mathfrak{g}_{0}=\left[\mathfrak{g}_{1}, \mathfrak{g}_{-1}\right] \cap \mathfrak{g}_{0} \not \supset 1$. Это доказывает, что $\mathfrak{g} \neq[\mathfrak{g}, \mathfrak{g}]$ и тем самым что сушествует инвариантный функционал $\theta$ на $\mathfrak{g}=\mathfrak{g l}(\lambda)$. Таким образом, функция $R=\left(1-e^{-2 t}\right) F_{\theta}(t)$ удовлетворяет уравнению

$$
T\left(\frac{d}{d t}\right) R(t)=0
$$

которое в явном виде записывается как

$$
\left(\lambda^{2}-\left(\frac{d}{d t}+1\right)^{2}\right) R(t)=0 .
$$

Если $\lambda \neq 0$, то решения имеют вид $R(t)=c_{1} e^{(\lambda-1) t}+c_{2} e^{-(\lambda+1) t}$, а из начального условия $R(0)=0$ следует, что

$$
F_{\theta}(t)=c \frac{e^{(\lambda-1) t}-e^{-(\lambda+1) t}}{1-e^{-2 t}}
$$

Отождествим скаляры со скалярными матрицами и нормируем функционал $\theta$ (след) естественным образом, т.е. полагая $\theta(1)=\lambda$. Такая нормировка фиксирует значение $c=1$.

Ясно, что при $\lambda=0$ характеристическое уравнение имеет много корней и

$$
F_{\theta}(t)=c \frac{t e^{-t}}{1-e^{-2 t}}
$$

Таким образом, $\mathfrak{l}(0)$ является бесконечномерной алгеброй Ли с $\infty$-мерным тождественным модулем. 


\section{4. АЛГЕБРЫ ЛИ $\mathfrak{g l}(\lambda) \mathbf{И ~}^{\mathfrak{l}^{-}}(\infty), \mathfrak{g l}^{+}(\infty), \mathfrak{g l}(\infty)$}

Пусть $V$ - векторное пространство с фиксированным базисом $v_{i}, i \in \mathbb{Z}$, пусть $V^{+}-$ подпространство, порожденное элементами $v_{i}$ при $i \geqslant 0$, а $V^{-}-$подпространство, порожденное элементами $v_{i}$ при $i<0$. Пусть $\mathfrak{g l}^{-}(\infty), \mathfrak{g l}^{+}(\infty)$ и $\mathfrak{g l}(\infty)$ - алгебры Ли линейных преобразований соответственно пространств $V^{-}, V^{+}$и $V$ таких, что их матрицы в фиксированных базисах имеют носитель на конечном числе диагоналей, параллельных главной (это число зависит от матришы).

На алгебре $\mathfrak{g l}(\infty)$ имеется (единственный) нетривиальный 2-коцикл

$$
c(A, B)=\operatorname{tr}([J, A] B), \quad \text { где } \quad J=\sum_{i \leqslant 0} E_{i i}-\sum_{i>0} E_{i i} .
$$

Обозначим через $\widehat{\mathfrak{g l}}(\infty)$ соответствуюшее центральное расширение; скобка в $\widehat{\mathfrak{g l}}(\infty)$ имеет вид

$$
[A, B]=A B-B A+\operatorname{tr}([J, A] B) \cdot z,
$$

где $z$ - новый центральный элемент.

В частности, для матричных элементов имеем

$$
\left[E_{i j}, E_{k l}\right]=\delta_{j k} E_{i l}-\delta_{i l} E_{k j}+\delta_{i l} \delta_{j k}(\kappa(i)-\kappa(j)) \cdot z,
$$

где $\kappa(i)=1$, если $i \leqslant 0$, и $\kappa(i)=0$ в других случаях.

Модули Хариш-Чандры $M^{\lambda, s}=\operatorname{Span}\left(v_{i}: i \in \mathbb{Z}\right)$ для любых $\lambda, s \in \mathbb{C}$ определяются над алгеброй $\mathfrak{s l}(2)$ как линейная оболочка элементов $v_{i}$, удовлетворяюших соотношениям

$$
\begin{aligned}
H v_{i} & =(s+2 i) v_{i}, \\
X v_{i} & =\frac{1}{2} \sqrt{(\lambda-s-2 i-1)(\lambda+s+2 i+1)} v_{i+1}, \\
Y v_{i} & =\frac{1}{2} \sqrt{(\lambda-s-2 i+1)(\lambda+s+2 i-1)} v_{i-1} .
\end{aligned}
$$

Легко видеть, что квадратичный оператор Казимира $\Omega$ действует на $M^{\lambda, s}$ как скалярный оператор умножения на $\left(\lambda^{2}-1\right) / 2$ и $M^{\lambda, s} \simeq M^{\lambda, s^{\prime}}$, если $s-s^{\prime} \in 2 \mathbb{Z}$.

Предположим, что $\lambda \in \mathbb{Z} \backslash\{0\}$. Тогда согласно [13] $M^{\lambda, s}$ неприводим, если $\lambda-s \notin$ $2 \mathbb{Z}+1$, и $M^{\lambda, s}=M_{+}^{\lambda, s} \oplus M_{-}^{\lambda, s}$, если $\lambda-s \in 2 \mathbb{Z}+1$, где $M_{+}^{\lambda, s}$ - неприводимый $\mathfrak{s l}(2)$-модуль с младшим весом $\lambda+1$, а $M_{-}^{\lambda, s}$ - неприводимый $\mathfrak{s l}(2)$-модуль со старшим весом $\lambda-1$; эти модули исчерпьвают все неприводимые $\mathfrak{s l}(2)$-модули с диагональным $H$-действием таким, что $\Omega$ действует как скалярньй оператор умножения на $\left(\lambda^{2}-1\right) / 2$.

Сушествует более прозрачная реализация модулей Хариш-Чандры. Положим

$$
X^{+}=x \frac{\partial}{\partial y}-2(\lambda-1) \frac{x}{y}, \quad X^{-}=y \frac{\partial}{\partial x} \quad\left(\text { т.е. } \quad H=x \frac{\partial}{\partial x}-y \frac{\partial}{\partial y}-(\lambda-1)\right)
$$

и $w_{i}=x^{a+i} y^{b-i}$. Тогда $\lambda=a+b+1, s=a-b$ и $w_{i}=v_{i}$ с точностью до скалярного множителя. 
Модуль $M^{\lambda, s}$ над $\mathfrak{g l}(\lambda)$ определяет вложение $\phi: \mathfrak{g l}(\lambda) \rightarrow \mathfrak{g l}(\infty)$. Поскольку $H^{2}(\mathfrak{g l}(\lambda))=0[5]$, существует подъем $\phi$ до гомоморфизма $\hat{\phi}: \mathfrak{g l}(\lambda) \rightarrow \widehat{\mathfrak{g l}}(\infty)$, который имеет вид $\hat{\phi}(u)=\phi(u)+\theta(u) \cdot z$, где $\theta-$ линейный функционал на $\mathfrak{g l}(\lambda)$ такой, что $\theta([u, v])=c(\phi(u), \phi(v))$ в терминах коцикла на $\mathfrak{g l}(\infty)$, который превращает эту алгебру в $\widehat{\mathfrak{g l}}(\infty)$. Заметим, что $\theta$ определяется однозначно с точностью до функционала, пропорционального следу на $\mathfrak{g l}(\lambda)$.

Следуюшая теорема описывает $\hat{\phi}$ более явным образом.

TеOPEмA 4.1. Пусть $\mathfrak{g}=\mathfrak{g l}(\lambda)$. Тогда $\hat{\phi}\left(\mathfrak{g}_{i}\right)=\phi\left(\mathfrak{g}_{i}\right)$ nрu $i \neq 0 u$

$$
\hat{\phi}\left(e^{t H}\right)=\phi\left(e^{t H}\right)-\frac{e^{s t}-e^{-(\lambda+1) t}}{1-e^{-2 t}} z .
$$

ДокАЗАТЕЛЬСТво.

$$
\begin{aligned}
\phi(X) & =\sum \alpha_{i} E_{i+1, i}, & \text { где } & \alpha_{i} & =\frac{1}{2} \sqrt{(\lambda-s-2 i-1)(\lambda+s+2 i+1)}, \\
\phi(H) & =\sum \gamma_{i} E_{i, i}, & \text { где } & \gamma_{i} & =s+2 i, \\
\phi(Y) & =\sum \beta_{i} E_{i-1, i}, & \text { где } & \beta_{i} & =\frac{1}{2} \sqrt{(\lambda-s-2 i+1)(\lambda+s+2 i-1)} .
\end{aligned}
$$

Следовательно,

$$
[J, \phi(X)]=\sum(\kappa(i)-\kappa(i+1)) \alpha_{i} E_{i+1, i}
$$

и $\phi\left(H^{k}\right) \phi(Y)=\sum \gamma_{i}^{k} \beta_{i+1} E_{i, i+1}$. Отсюда

$$
[J, \phi(X)] \phi\left(H^{k}\right) \phi(Y)=\sum \gamma_{i}^{k} \beta_{i+1} \alpha_{i}(\kappa(i)-\kappa(i+1)) E_{i+1, i+1}
$$

и

$$
\begin{aligned}
c\left(\phi(X), \phi\left(H^{k} Y\right)\right) & =\operatorname{tr}\left([J, \phi(X)] \phi\left(H^{k} Y\right)\right)=\sum \gamma_{i}^{k} \beta_{i+1} \alpha_{i}(\kappa(i)-\kappa(i+1))= \\
& =\gamma_{0}^{k} \beta_{1} \alpha_{0}=\frac{1}{4} s^{k}\left(\lambda^{2}-(s+1)^{2}\right)=T(s) s^{k} .
\end{aligned}
$$

Таким образом,

$$
\begin{aligned}
T\left(\frac{d}{d t}\right)\left(e^{s t}\right) & =T(s) e^{s t}=\sum_{k \geqslant 0} \frac{T(s) s^{k}}{k !} t^{k}=\sum_{k \geqslant 0} \frac{c\left(\phi(X), \phi\left(H^{k} Y\right)\right)}{k !} t^{k}= \\
& =\sum_{k \geqslant 0} \frac{\theta\left(\left[X, H^{k} Y\right]\right)}{k !} t^{k}=\sum_{k \geqslant 0} \frac{\theta\left(X H^{k} Y-H^{k} Y X\right)}{k !} t^{k}= \\
& =\sum_{k \geqslant 0} \frac{\theta\left(T(H-2)(H-2)^{k}-H^{k} T(H)\right)}{k !} t^{k}=T\left(\frac{d}{d t}\right)\left(\left(e^{-2 t}-1\right) \theta\left(e^{t H}\right)\right) .
\end{aligned}
$$

Следовательно,

$$
T\left(\frac{d}{d t}\right)\left(\left(e^{-2 t}-1\right) \theta\left(e^{t H}\right)-e^{s t}\right)=0
$$




$$
\left(e^{-2 t}-1\right) \theta\left(e^{t H}\right)= \begin{cases}e^{s t}+c_{1} e^{-(\lambda+1) t}+c_{2} e^{(\lambda-1) t}, & \text { если } \lambda \neq 0 \\ e^{s t}+c_{1} e^{-t}+c_{2} t e^{-t}, & \text { если } \lambda=0\end{cases}
$$

В обоих случаях, вычитая слагаемое, пропорциональное следу, получим

$$
\left(e^{-2 t}-1\right) \theta\left(e^{t H}\right)=e^{s t}-e^{-(\lambda+1) t}
$$

Теорема 4.1 доказана.

Рассмотрим алгебру Ли $\mathfrak{g l}(\infty)$ над алгеброй усеченных многочленов. Пусть $R_{m}=$ $\mathbb{C}[\varepsilon]$, где $\varepsilon^{m+1}=0$. Расширяя модуль $M^{\lambda, s}$ до модуля $M_{R_{m}}^{\lambda, s}$ над $R_{m}$, т.е. считая, что $s \in R_{m}$, можно определить действие алгебры $\mathfrak{s l}(2)$ теми жее формулами. Если $v_{i}-$ исходный базис, то в качестве фиксированного базиса в расширенном модуле $M_{R_{m}}^{\lambda, s}$ мы возьмем $\varepsilon^{j} v_{i}$ для всех $i \in \mathbb{Z}$ и $j=1, \ldots, m$. Заметим, что в этом базисе в $M_{R_{m}}^{\lambda, s}$ действие элемента $H$ недиагонально.

Алгебра $\mathfrak{g l}\left(\infty ; R_{m}\right)$ и ее подалгебры $\mathfrak{g l}^{\ddagger}\left(\infty ; R_{m}\right)$ определены естественным образом. Центральное расширение $\widehat{\mathfrak{g l}}\left(\infty ; R_{m}\right)$ определяется теми же формулами.

Рассматривая $s+\varepsilon$ вместо $s \in \mathbb{C}$, получим следующее следствие теоремы 4.1.

СледСТвИЕ 4.1. Гомоморфизм $\hat{\phi}: \mathfrak{g l}(\lambda) \rightarrow \widehat{\mathfrak{g l}}\left(\infty ; R_{m}\right)$, индуцированный вложсением $\phi: \mathfrak{g l}(\lambda) \rightarrow \mathfrak{g l}\left(\infty ; R_{m}\right)$, удовлетворяет равенству $\hat{\phi}\left(\mathfrak{g l}(\lambda)_{i}\right)=\phi\left(\mathfrak{g l}(\lambda)_{i}\right)$ при $i \neq 0 u$

$$
\hat{\phi}\left(e^{t H}\right)=\phi\left(e^{t H}\right)-\left(\frac{e^{s t}-e^{-(\lambda+1) t}}{1-e^{-2 t}}+\frac{e^{s t}}{1-e^{-2 t}} \sum_{j=1}^{m} \frac{\varepsilon^{j} t^{j}}{j !}\right) z .
$$

Заметим, что мы "вынуждены" рассматривать центральное расширение $\widehat{\mathfrak{g l}}(\infty)$ для описания квазиконечных модулей над $\mathfrak{g l}(\lambda)$, поскольку $\widehat{\mathfrak{g l}}(\infty)$ имеет большее число квазиконечных представлений, чем $\mathfrak{g l}(\infty)$, и рассмотрение только $\mathfrak{g l}(\infty)$-модулей оказывается недостаточным.

Следуюшие утверждения описывают условия на старший вес неприводимого модуля, которые являются необходимыми и достаточными, для того чтобы модуль был квазиконечным.

Обозначим через $\mathfrak{g l}_{f}(\infty)$ и $\mathfrak{g l} \mathfrak{l}_{f}^{ \pm}(\infty)$ соответственно подалгебры в $\mathfrak{g l}(\infty)$ и $\mathfrak{g l}^{ \pm}(\infty)$, состояшие из матриц с конечным носителем. Пусть $\mathfrak{h}$ обозначает подалгебру Картана любой из этих алгебр Ли; рассмотрим ее как порожденную диагональными элементами $E_{i i}$. Заметим, что ограничение вложения $\hat{\phi}: \mathfrak{g l}_{f}(\infty) \rightarrow \widehat{\mathfrak{g l}}(\infty)$ на $\mathfrak{h}$ имеет вид $E_{i i} \mapsto \phi\left(E_{i i}\right)+\kappa(i) z$, где $\phi$ - естественное вложение $\mathfrak{g l}_{f}(\infty)$ в $\mathfrak{g l}(\infty)$. Если $\theta$ - вес модуля (со старшим весом) над одной из указанных алгебр Ли $(\mathfrak{g l}(\infty)$ и ее подалгебры, а также $\mathfrak{g l}_{f}(\infty)$ и ее подалгебры), мы можем найти координаты $\theta_{i}=\theta\left(E_{i i}\right)$ веса $\theta$, рассматривая $\mathfrak{h}$ указанным выше способом.

ПРЕДЛОЖЕНИЕ 4.1. $\mathfrak{g l}_{f}(\infty)$-Модуль со стариим весом $\Lambda$ является квазиконечным, если и только если существует лишь конечное число различных координат $\lambda_{i}=\Lambda\left(E_{i i}\right)$. 
ДокАЗАТЕльСтво. Пусть $V$ квазиконечен и генерируется вектором старшего веса $v$. Поскольку $\operatorname{dim} V_{-1}<\infty$ для конечного множества $I V_{-1}=\operatorname{Span}\left(E_{i+1, i} v\right)_{i \in I}$. Пусть $k \notin I$ и $k+1 \notin I$. Тогда

$$
E_{k+1, k} v=\sum_{i \in I} \alpha_{i} E_{i+1, i} v
$$

поэтому $E_{k, k+1}\left(E_{k+1, k} v\right)=0$ или, что эквивалентно, $\left(E_{k, k}-E_{k+1, k+1}\right) v=0$, т.е. $\lambda_{k}=\lambda_{k+1}$.

Обратное утверждение очевидно.

ПРЕДЛОЖЕНИЕ 4.2. $\mathfrak{g l}(\infty)-$ Модуль $V$ со старшим весом $\Lambda$, әде $\lambda_{i}=\lambda\left(E_{i i}\right)$, является квазиконечным, если и только если существует лишь конечное число ненулевых координат $\lambda_{i}$, где $\lambda_{i}=\Lambda\left(E_{i i}\right)$.

ДокАЗАтЕЛЬСтво. Рассматривая $V$ как $\mathfrak{g l}_{f}(\infty)$-модуль, мы видим, что имеется только конечное число различных координат $\lambda_{i}$, откуда следует, что $\lambda_{k}=\lambda_{+}$и $\lambda_{-k}=$ $\lambda_{-}$при достаточно больших $k$. Поэтому $E_{k, k+1}\left(E_{k+1, k} v\right)=0$ и, поскольку $V$ является неприводимым, $E_{k+1, k} v=0$ для всех, кроме конечного числа, $k$.

Пусть

$$
A=\sum_{i \in \mathbb{Z}} E_{i, i+1}, \quad B_{k}=\sum_{i \geqslant k} E_{i+1, i} .
$$

Тогда

$$
\left[A, B_{k}\right]=\sum_{i \geqslant k} E_{i, i}-\sum_{i \geqslant k} E_{i+1, i+1}=E_{k, k}
$$

Следовательно,

$$
\begin{aligned}
{\left[A, B_{k}\right] v } & =E_{k, k} v=\lambda_{k} v=A B_{k} v-B_{k} A v=A B_{k} v=A\left(\sum_{i \geqslant k, i \in I} E_{i+1, i} v\right)= \\
& =\sum_{i \geqslant k, i \in I}\left[A, E_{i+1, i}\right] v=\sum_{i \geqslant k, i \in I}\left(E_{i, i}-E_{i+1, i+1}\right) v= \\
& =\left(\sum_{i \geqslant k, i \in I}\left(\lambda_{i}-\lambda_{i+1}\right)\right) v=\left(\lambda_{k}-\lambda_{j}\right) v
\end{aligned}
$$

где $j$ - наибольший индекс в конечном множестве $I$. Поэтому $\lambda_{k}=\lambda_{k}-\lambda_{j}$ и $\lambda_{j}=0$.

Аналогично, если

$$
C_{k}=\sum_{i \leqslant k} E_{i+1, i}
$$

TO

$$
\left[A, C_{k}\right]=\sum_{i \leqslant k} E_{i, i}-\sum_{i \leqslant k} E_{i+1, i+1}=E_{k+1, k+1}
$$

Следовательно,

$$
\begin{aligned}
{\left[A, C_{k}\right] v } & =\lambda_{k+1} v=A C_{k} v=A\left(\sum_{i \leqslant k} E_{i+1, i}\right) v=\sum_{i \leqslant k}\left[A, E_{i+1, i}\right] v= \\
& =\sum_{i \leqslant k}\left(E_{i, i}-E_{i+1, i+1}\right) v=\sum_{i \leqslant k}\left(\lambda_{i}-\lambda_{i+1}\right) v=\left(\lambda_{j}-\lambda_{k+1}\right) v .
\end{aligned}
$$


Таким образом, $\lambda_{j}=0$ для достаточно больших $j$. Обратное утверждение следует из предложения 4.1, что и требовалось доказать.

ПрЕДЛОЖенИЕ 4.3. $\widehat{\mathfrak{g l}}(\infty)$-Модуль $V$ со стариим весом $(\Lambda, c)$, әде $c$ - значение чентрального заряда на $z$, квазиконечен, если и только если имеется лишь конечное число ненулевых координат $\lambda_{i}$, где $\lambda_{i}=\Lambda\left(E_{i i}\right)$.

ДокАЗАТЕльСтво. Как и в доказательстве предложения 4.2, покажем, что имеется только конечное число различных координат $\lambda_{i}$ и $E_{k+1, k} v \neq 0$ для конечного числа значений $k$.

Пусть

$$
A=\sum_{i \in \mathbb{Z}} E_{i, i+1}, \quad J=\sum_{i \leqslant 0} E_{i, i} .
$$

Тогда $[A, J]=E_{01}$, следовательно, $c(A, B)=\operatorname{tr}\left(E_{01} B\right)$ для любых $B \in \mathfrak{g l}(\infty)$ и, таким образом, $c\left(A, B_{k}\right)=\kappa(k)$. Имеем

$$
\begin{aligned}
{\left[A, B_{k}\right] v } & =E_{k, k} v=\left(E_{k, k}+\kappa(k) z\right) v=A B_{k} v=A\left(\sum_{i \geqslant k, i \in I} E_{i+1, i} v\right)= \\
& =\sum_{i \geqslant k, i \in I}\left[A, E_{i+1, i}\right] v=\sum_{i \geqslant k, i \in I}\left(E_{i, i}-E_{i+1, i+1}+\delta_{i 0} z\right) v= \\
& =\kappa(k) z+\left(\sum_{i \geqslant k, i \in I}\left(\lambda_{i}-\lambda_{i+1}\right)\right) v=\kappa(k) c v+\left(\lambda_{k}-\lambda_{+}\right) v=\kappa(k) c+\left(\lambda_{k}\right) v
\end{aligned}
$$

поэтому $\lambda_{+}=0$.

Для $C_{k}=\sum_{i \leqslant k} E_{i+1, i}$ имеем $c\left(A, C_{k}\right)=\kappa(-k)$, следовательно,

$$
\begin{aligned}
{\left[A, C_{k}\right] v } & =\left(-E_{k+1, k+1}-\kappa(-k)\right) v=\left(-\lambda_{k+1}-\kappa(-k) c\right) v=A C_{k} v= \\
& =A \sum_{i \leqslant k} E_{i+1, i} v=\sum_{i \leqslant k}\left[A, E_{i+1, i}\right] v=\sum_{i \leqslant k}\left(E_{i, i}-E_{i+1, i+1}+\delta_{i 0} z\right) v= \\
& =\left(\kappa(-k) c+\lambda_{-}-\lambda_{k+1}\right) v .
\end{aligned}
$$

Таким образом, $\lambda_{-}=0$, что и требовалось доказать.

ТеОРема 4.2. Пусть $\mathfrak{g}$ - одна из алгебр Ли $\mathfrak{g l}\left(\infty ; R_{m}\right)$ или $\mathfrak{g l}^{ \pm}\left(\infty ; R_{m}\right)$, или их вариантов с крышками; g-модуль $V$ со стариим весом $\theta$, заданный своими координатами $\theta_{i j}=\left(\varepsilon^{j} E_{i i}\right)$, является квазиконечным, если и только если имеется лишь конечное число ненулевых координат $\theta_{i j}$.

ДокАЗАТЕЛЬСТВО аналогично доказательствам предложений 4.1, 4.2 и теоремы 4.1.

Пусть $V$ - один из модулей $M_{R_{m}}^{\lambda, s}$ или, если $M_{R_{m}}^{\lambda, s}$ приводим, один из его неприводимыхх подмодулей. Получаем гомоморфизм алгебры $\mathfrak{g l}(\lambda)$ в $\mathfrak{g}=\mathfrak{g l}\left(\infty ; R_{m}\right)$ или $\mathfrak{g l} \mathfrak{l}^{ \pm}\left(\infty ; R_{m}\right)$. Пусть $\theta$ - линейньй функционал, удовлетворяюший условиям теоремы 4.2. Мы можем рассматривать $\theta$ как линейный функционал на подалгебре Картана алгебры $\mathfrak{g l}(\lambda)$. Вычислим соответствуюшую производяшую функцию. 
Теорема 4.3. Производящая функиия $F_{\theta}(t)$ для функиионала $\theta$ имеет вид:

I. ДЛя $M_{R_{m}}^{\lambda, s}$

$$
\begin{aligned}
F_{\theta}(t)= & \frac{\sum_{i \in \mathbb{Z}} e^{(s-2 i) t} \sum_{j=0}^{m} \frac{\left(\theta_{i j}-\theta_{i-1, j}\right) t^{j}}{j !}}{1-e^{-2 t}}- \\
& -\left(\frac{e^{s t}-e^{-(\lambda+1) t}}{1-e^{-2 t}} c+\frac{e^{s t}}{1-e^{-2 t}} \sum_{j=1}^{m} \frac{c_{j}}{j !}\right) .
\end{aligned}
$$

II. Для модуля со стариим весом $\lambda-1$

$$
F_{\theta}(t)=\frac{\sum_{i \in \mathbb{Z}} e^{(\lambda-2 i-1) t} \sum_{j=0}^{m} \frac{\left(\theta_{i j}-\theta_{i-1, j}\right) t^{j}}{j !}}{1-e^{-2 t}} .
$$

III. Для модуля со стариим весом $-\lambda-1$

$$
F_{\theta}(t)=\frac{\sum_{i \in \mathbb{Z}} e^{(-\lambda-2 i-1) t} \sum_{j=0}^{m} \frac{\left(\theta_{i j}-\theta_{i-1, j}\right) t^{j}}{j !}}{1-e^{-2 t}} .
$$

IV. Для модуля с младиим весом $\lambda+1$

$$
F_{\theta}(t)=\frac{\sum_{i \in \mathbb{Z}} e^{(\lambda+2 i+1) t} \sum_{j=0}^{m} \frac{\left(\theta_{i j}-\theta_{i-1, j}\right) t^{j}}{j !}}{1-e^{-2 t}} .
$$

V. Для модуля с младиим весом $1-\lambda$

$$
F_{\theta}(t)=\frac{\sum_{i \in \mathbb{Z}} e^{(1-\lambda+2 i) t} \sum_{j=0}^{m} \frac{\left(\theta_{i j}-\theta_{i-1, j}\right) t^{j}}{j !}}{1-e^{-2 t}} .
$$

ДОКАЗАТЕЛЬСтво. Докажем только утверждение I, остальные доказываются аналогично. Пусть $\phi$-гомоморфизм, определяюший $\mathfrak{g l}(\lambda)$-действие на $M^{\lambda, s}$. Тогда

$$
\phi(H)=\sum_{i \in \mathbb{Z}}(s+\varepsilon-2 i) E_{i i},
$$

следовательно,

$$
\phi\left(e^{t H}\right)=\sum_{i \in \mathbb{Z}} e^{(s+\varepsilon-2 i) t} E_{i i}
$$

И

$$
\begin{aligned}
\phi\left(\left(1-e^{-2 t}\right) e^{t H}\right) & =\phi\left(e^{t H}\right)-\phi\left(e^{t(H-2)}\right)=\sum_{i \in \mathbb{Z}} e^{(s+\varepsilon-2 i) t}\left(E_{i i}-E_{i-1, i-1}\right)= \\
& =\sum_{i \in \mathbb{Z}} e^{(s-2 i) t} \sum_{j=0}^{m} \varepsilon^{j}\left(E_{i i}-E_{i-1, i-1}\right) \frac{t^{j}}{j !} .
\end{aligned}
$$

Поэтому

$$
\left(1-e^{-2 t}\right) F_{\theta}(t)=\theta\left(\phi\left(e^{t H}\right)-\phi\left(e^{t(H-2)}\right)\right)=\sum_{i \in \mathbb{Z}} e^{(s-2 i) t} \sum_{j=0}^{m} \frac{\theta_{i j}-\theta_{i-1, j}}{j !} t^{j} .
$$

Поскольку только конечное число координат $\theta_{i j}$ не равно нулю, все суммы в действительности являются конечными, что и требовалось доказать. 


\section{5. КВАЗИКОНЕЧНЫЕ МОДУЛИ НАД АЛГЕБРОЙ $\mathfrak{g l}(\lambda)$}

Ниже мы покажем, что не все квазиконечные модули над $\mathfrak{g l}(\lambda)$ могут быть представлены в каноническом виде, как это было сделано для $W_{1+\infty}$ в работе [14].

Пусть $\mathfrak{g l}^{\mathrm{hol}}(\lambda)$ - голоморфное пополнение алгебры $\mathfrak{g l}(\lambda)$, т.е. алгебра функций от $H$, голоморфных на всей комплексной прямой $\mathbb{C}$, и пусть $\mathfrak{g l}_{i}^{\text {hol }}(\lambda)=\left\{f(H) X^{i}\right\}$ при $i>0$, $\mathfrak{g g h}_{i}^{\mathrm{hol}}(\lambda)=\left\{f(H) Y^{-i}\right\}$ при $i<0$ и $f \in \mathfrak{g l}_{0}^{\mathrm{hol}}(\lambda)$. Соотношения в пополненной алгебре следуют из уравнений (1.7) и (1.10):

$$
X f(H)=f(H-2) X, \quad Y f(H)=f(H+2) Y, \quad X Y=\frac{1}{4}\left(\lambda^{2}-(H-1)^{2}\right) .
$$

Следуюшее предложение доказьвается аналогично соответствуюшему утверждению работы [14].

ПРЕДЛОЖенИЕ 5.1. Пусть $V$ - квазиконечный $\mathfrak{g l}(\lambda)$-модуль. Тогда $\mathfrak{g l}(\lambda)$-действие естественно продолэсается до $\mathfrak{g h}_{i}^{\mathrm{hol}}(\lambda)$-действия при $i \neq 0$.

Введем на $\mathbb{C}$ соотношение эквивалентности, положив

$$
[s]= \begin{cases}s+2 \mathbb{Z}, & \text { если } s+\lambda \notin 2 \mathbb{Z}+1, \\ s-2 \mathbb{Z}_{+}, & \text {если } s= \pm \lambda+1, \\ s+2 \mathbb{Z}_{+}, & \text {если } s= \pm \lambda-1 .\end{cases}
$$

Поставим в соответствие каждому классу $[s]$ неприводимый $\mathfrak{s l}(2)$-модуль $M^{s}$ с диагональным действием генератора $H$, множество собственных значений которого совпадает с $[s]$; обозначим через $\mathfrak{g l}_{s}(\infty)$ алгебру Ли линейных преобразований в $M^{s}$ с конечным числом ненулевых диагоналей в $H$-диагональном базисе.

Следуюшая теорема аналогична и доказывается так же, как соответствуюшая теорема в работе [14].

Теорема 5.1. Пусть $\left[s_{i}\right]-$ различные, а $m_{i}$ - неотрицательные иелье числа. Пусть

$$
\phi: \mathfrak{g l}(\lambda) \longrightarrow \mathfrak{g}=\bigoplus_{i=1}^{k} \mathfrak{g l}_{s_{i}}\left(\infty ; R_{m_{i}}\right)
$$

определяется модулем $\bigoplus_{i=1}^{k} M_{R_{m_{i}}}^{s_{i}}$. Тогда для любого квазиконечного g-модуля $V$ любой его $\mathfrak{g l}(\lambda)$-подмодуль является $\mathfrak{g}$-подмодулем. В частности, если $V$ неприводим как $\mathfrak{g}$-модуль, он неприводим и как $\mathfrak{g l}(\lambda)$-модуль.

Опишем, следуя работе [14], структуру квазиконечных $\mathfrak{g l}(\lambda)$-модулей. Пусть $\theta$ - старший вес квазиконечного $\mathfrak{g l}(\lambda)$-модуля и

$$
F_{\theta}(t)=\frac{R(t)}{1-e^{-2 t}},
$$

где $R(t)=\sum r_{i}(t) e^{s_{i} t}$ - соответствуюший формальный степенной ряд (см. раздел 3$)$. Многочлены $r_{i}(t)$ называются кратностями. 
Для каждого $s$ обозначим через $R_{s}(t)$ сумму всех квазимногочленов с показателями из класса $[s]$. Тогда $R(t)=\sum R_{s}(t)$, где сумма идет по представителям различных классов эквивалентности. Пусть $R_{s}(t)=\sum r_{i s}(t) e^{(s-2 i) t}$. Из определений легко получить следующие свойства квазимногочленов:

a) $R(0)=0$;

б) сумма всех кратностей $R_{\lambda+1}(t)$, а также сумма всех кратностей $R_{-\lambda+1}(t)$ равны нулю;

в) сумма всех кратностей $R_{\lambda-1}(t)$, а также сумма всех кратностей $R_{-\lambda-1}(t)$ равны константе.

Введем обозначение $\Lambda=[\lambda-1] \cap[\lambda+1] \cap[-\lambda-1] \cap[-\lambda+1]$. Квазимногочлену $R$, удовлетворяющему свойствам “а" - “в”, поставим в соответствие $\mathfrak{g l}(\lambda)$-модуль $V(s)$ следующим образом:

1) если $s \notin \Lambda$, то $V(s)$ - неприводимый $\widehat{\mathfrak{g l}}(\infty)$-модуль с центральными зарядами

$$
c_{j}=-\sum_{i \in I} r_{i}^{(j)}(0),
$$

где $j=0, \ldots, \max \left(\operatorname{deg} r_{i}\right)$, а остальные координаты старшего веса равны

$$
\theta_{i j}=\sum_{l \leqslant i}\left(r_{l}^{(j)}(0)+\delta_{l 0} c_{j}\right)
$$

2) если $s \notin[\lambda-1] \cap[-\lambda-1]$ и

$$
R_{s}=\sum_{i} r_{i}(y) e^{s-2 i}
$$

положим

$$
c=-\sum_{i \in I} r_{i}(0), \quad \theta_{i j}=\sum_{l \leqslant i}\left(r_{l}^{(j)}(0)+\delta_{l 0} \delta_{j 0} c_{j}\right),
$$

тогда $V(s)$ - соответствуюший $\mathfrak{g l}^{-}(\infty)$-модуль;

$3)$ если $s \notin[-\lambda+1] \cap[\lambda+1]$, положим

$$
\theta_{i j}=\sum_{l \leqslant i} r_{l}^{(j)}(0)
$$

тогда $V(s)$ - соответствующий $\mathfrak{g l}^{+}(\infty)$-модуль.

Теорема 5.2. Пусть квазимногочлен

$$
R=\sum_{i=1}^{k} R_{s_{i}}(t)
$$

разложен по различным классам әквивалентности показателей; пусть $R$ удовлетворяет перечисленным выце свойствам "а"- "в". Тогда неприводимый квазиконечный модуль со старшим весом $R(t)$ изоморфен модулю

$$
V=V\left(s_{1}\right) \otimes \cdots \otimes V\left(s_{k}\right) \otimes(\alpha \operatorname{tr}),
$$

где $\alpha \operatorname{tr}$ - неприводимый 1-мерный модуль, соответствующий следу на $\mathfrak{g l}(\lambda)$, а модули $V\left(s_{i}\right)$ построены так, как это описано выше. 
ДокАЗАтЕльСтво. Согласно теореме 4.3 модуль $V$ неприводим как $\mathfrak{g l}(\lambda)$-модуль. Поэтому достаточно показать, что ограничение старшего веса этого модуля на $\mathfrak{g l}(\lambda)$ равно $R(t) /\left(1-e^{-2 t}\right)$. Пусть $s=s_{i}-$ один из показателей такой, что $s \notin \Lambda$. Тогда предложение 5.1 означает, что сушествует старший вес $\theta_{s}$ для $\widehat{\mathfrak{g l}}\left(\infty ; R_{m}\right)$, производящая функция которого равна

$$
\frac{R_{s}(t)+c_{s} e^{-(\lambda+1) t}}{1-e^{-2 t}}
$$

где $c_{s}=-R_{s}(0)$

Если $s \in \Lambda$, то же предложение означает, что производящая функция равна $R_{s}(t) /\left(1-e^{-2 t}\right)$, где сумма всех показателей $R_{s}$ равна нулю.

Наконец, производяшая функция следа есть

$$
\alpha \frac{e^{(\lambda-1) t}-e^{-(\lambda+1) t}}{1-e^{-2 t}} .
$$

Поэтому для нашего модуля $V$ производяшая функция имеет вид $R(t) /\left(1-e^{-2 t}\right)$, где при соответствуюшем выборе $c_{s}$

$$
\begin{aligned}
R(t)= & \sum_{s \notin \Lambda}\left(R_{s}(t)+c_{s} e^{-(\lambda+1) t}\right)+ \\
& +R_{\lambda+1}(t)+R_{-\lambda+1}(t)+R_{-\lambda-1}^{\prime}(t)+R_{\lambda-1}^{\prime}(t)+\alpha\left(e^{(\lambda-1) t}-e^{-(\lambda+1) t}\right)= \\
= & \sum_{s \notin \Lambda} R_{s}(t)+R_{\lambda+1}(t)+R_{-\lambda+1}(t)+ \\
& +\left(R_{-\lambda-1}^{\prime}(t)-\alpha e^{-(\lambda+1) t}\right)+\left(R_{\lambda-1}^{\prime}(t)+\alpha e^{(\lambda-1) t}\right)=\sum_{s \notin \Lambda} R_{s}(t),
\end{aligned}
$$

что требовалось доказать.

\section{6. УНИТАРНЫЕ МОДУЛИ НАД АЛГЕБРОЙ $\mathfrak{g l}(\lambda)$}

Напомним, что антиинволюиия на алгебре Ли $\mathfrak{g}$ над $\mathbb{C}$ - это $\mathbb{R}$-линейное отображение $\omega: \mathfrak{g} \rightarrow \mathfrak{g}$ такое, что

$$
\omega(\alpha x)=\bar{\alpha} \omega(x), \quad \omega([x, y])=[\omega(x), \omega(y)], \quad \omega^{2}=\mathrm{id}
$$

для любых $\alpha \in \mathbb{C}_{\text {и }} x, y \in \mathfrak{g}$.

Задав антиинволюцию $\omega$, мы можем снабдить модуль $V^{*}$, дуальный к $\mathfrak{g}$-модулю $V$, другой структурой g-модуля, а именно

$$
(x l)(v)=l(\omega(x) v) \quad \text { для любых } l \in V^{*}, v \in V \text { и } x \in \mathfrak{g} .
$$

В частности, если $\mathfrak{g}=\mathfrak{g}_{-} \oplus \mathfrak{g}_{0} \oplus \mathfrak{g}_{+}$и $\omega$ переставляет $\mathfrak{g}_{-}$и $\mathfrak{g}_{+}$, то $\mathfrak{g}$-гомоморфизм $M \rightarrow M^{*}$ является хорошо определенным; здесь $M$ - модуль Верма с вектором старшего веса $v$, а структура $\mathfrak{g}$-модуля на $M^{*}$ задается уравнением (6.1).

Действительно, пусть $v^{*} \in M^{*}$, причем $v^{*}(v)=1$ и $v^{*}(u)=0$ для любых $u$ с весом, меньшим чем вес $v$. Следовательно, если $x \in \mathfrak{g}_{+}$, то $\left(x v^{*}\right)(u)=v^{*}(\omega(x) u)=0$ и, таким образом, $v^{*}$ также является вектором старшего веса. Если $\theta(\omega(H))=\overline{\theta(H)}$, где 
$\theta$ - вес вектора $v$, то вес вектора $v^{*}$ также равен $\theta$. Поэтому сушествует $\mathfrak{g}$-изоморфизм $M \rightarrow M^{*}$ или эрмитова $\mathfrak{g}$-инвариантная форма $\langle\cdot, \cdot\rangle$ на $M$. Если $\langle\cdot, \cdot\rangle$ положительно определена, модуль $M$ называется унитарныц.

В данном разделе мы укажем условия на старший вес квазиконечного $\mathfrak{g l}(\lambda)$-модуля, для того чтобы этот модуль был унитарен. Сначала опишем автоморфизмы алгебры $\mathfrak{g l}(\lambda)$.

Как хорошо известно, описание автоморфизмов алгебры функций $\mathcal{F}$ (в особенности многочленов) представляет собой сложную задачу. Столь же сложна и задача описания автоморфизмов алгебры Ли $\mathfrak{d e r}(\mathcal{F})$ дифференцирований алгебры $\mathcal{F}$. Для уменшшения числа автоморфизмов до разумного значения представляется естественным рассматривать только внешние автоморфизмы, т.е. классы автоморфизмов по модулю группы внутренних автоморфизмов. Какие автоморфизмы следует рассматривать как внутренние? Для алгебры $\mathfrak{d e r}(\mathcal{F})$ естественным образом подходят автоморфизмы, индуцированные автоморфизмами алгебры $\mathcal{F}$. Аналогично можно сказать, что автоморфизм $\phi$ алгебры $\mathfrak{g l}(\lambda)$ является внутренним, если он является также автоморфизмом ассоциативной алгебры $\mathfrak{A}_{\lambda}$.

Распространим это определение на алгебру $L U_{\mathfrak{g}}(\lambda)$ и назовем автоморфизм этой алгебры внутренним, если он также является автоморфизмом ассоциативной алгебры $U_{\mathfrak{g}}(\lambda)$.

Напомним, что линейное отображение $\phi: \mathfrak{g} \rightarrow \mathfrak{g}$ есть антиавтоморфизм алгебры Ли $\mathfrak{g}$, если $\phi([x, y])=[\phi(y), \phi(x)]$. Для ассоциативных алгебр определение антиавтоморфизма аналогично. Например, отображение $t$ такое, что $\left.{ }^{t}\right|_{\mathfrak{g}}=-\mathrm{id}$, называется главныцм антиавтоморфизмом алгебры Ли $\mathfrak{g}$. Главньй антиавтоморфизм алгебры g можно продолжить до антиавтоморфизма ассоциативной алгебры $U(\mathfrak{g}):{ }^{t}(x \otimes y)={ }^{t} y \otimes{ }^{t} x$. Ясно, что $t$ сохраняет элементы Казимира.

Теорема 6.1. Группа внешних автоморфизмов алгебры $\mathfrak{g l}(\lambda)$ изоморфна группе $\mathbb{Z} / 2$ и порождена классом әлемента $-t$, әде $-t: x \mapsto-{ }^{t} x$.

ДокАЗАтЕльСтво. Пусть $\psi \in \operatorname{Aut}(\mathfrak{g l}(\lambda))$. Тогда $\psi(Y), \psi(H)$ и $\psi(X)$ порождают алгебру Ли, изоморфную $\mathfrak{s l}(2)$. Более того, они порождают $\mathfrak{A}_{\lambda}$, потому что вес $\psi(X)^{n}$ равен $2 n$ относительно веса $\psi(H)$. Следовательно, сушествует сюръективньй гомоморфизм

$$
\tau: U(\mathfrak{s l}(2)) \rightarrow \mathfrak{A}_{\lambda}, \quad X \mapsto \psi(X), \quad H \mapsto \psi(H), \quad Y \mapsto \psi(Y)
$$

Поскольку $\tau(\Omega) \in \mathbb{C}$, то сушествует такое $\mu \in \mathbb{C}$, при котором $\tau(\Omega)=\left(\mu^{2}-1\right) / 2$. Поэтому мы получаем сюръективный гомоморфизм и, следовательно, изоморфизм $\mathfrak{A}_{\mu} \rightarrow \mathfrak{A}_{\lambda}$. В работе [6] Диксмье доказал, что это возможно, только если $\lambda^{2}=\mu^{2}$. Таким образом, можно считать, что $\lambda=\mu$ и $\tau$ является автоморфизмом. Пусть $\psi_{1}=\tau^{-1} \psi$, тогда $\left.\psi_{1}\right|_{\mathfrak{s l}(2)}=\mathrm{id}$.

Следовательно, $\psi_{1}$ является автоморфизмом алгебры $\mathfrak{g l}(\lambda)$, рассматриваемой как $\mathfrak{s l}(2)$-модуль. Поэтому $\left.\psi_{1}\right|_{L^{2 i}}=c_{i} \in \mathbb{C}$. Поскольку $L^{2}$ и $L^{4}$ порождают алгебру Ли $\mathfrak{g l}(\lambda)$, можно заключить, что $c_{i}=c_{2}^{i-1}$ для любых $i \geqslant 1$. Более того, из условия $\left[L^{4}, L^{4}\right] \supset L^{2}$ следует, что $c_{2}^{2}=1$. Поэтому $c_{2}= \pm 1$. Если $c_{2}=1$, то $\psi_{1}=\mathrm{id}$, а если $c_{2}=-1$, то $\psi_{1}=\phi$. Теорема доказана. 
Теорема 6.2. Если $\lambda^{2} \notin \mathbb{R}$, то $\mathfrak{g l}(\lambda)$ не имеет вещественных форм, т.е. инволютивных антилинейных автоморфизмов.

ДокАЗАТЕЛЬСТвО. Пусть $\omega$ - инволютивный антилинейный автоморфизм алгебры $\mathfrak{g l}(\lambda)$. Тогда $\omega(X), \omega(H)$ и $\omega(Y)$ порождают $\mathfrak{A}_{\lambda}$. Следовательно, сушествует сюръективный гомоморфизм

$$
\tau: U(\mathfrak{s l}(2)) \rightarrow \mathfrak{A}_{\lambda}, \quad \tau=\text { id } \quad \text { на } X, H, Y,
$$

откуда мы получаем изоморфизм $\tau: \mathfrak{A}_{\mu} \rightarrow \mathfrak{A}_{\lambda}$. Согласно работе [6] это возможно, только если $\lambda^{2}=\mu^{2}$. Следовательно, $\lambda=\mu$ и $\tau$ является автоморфизмом. Тогда $\omega_{1}=\tau^{-1} \omega-$ антилинейный автоморфизм $\mathfrak{g l}(\lambda)$ такой, что

$$
\omega_{1}(X)=X, \quad \omega_{1}(H)=H, \quad \omega_{1}(Y)=Y .
$$

Положим $z_{i}=(\operatorname{ad} X)^{i}\left(Y^{2}\right)$, в частности $z_{0}=z$. Тогда $\operatorname{Span}\left(z_{i}: i=1, \ldots, 4\right)$ образует базис $L^{4}$ и, если $\omega_{1}\left(Y^{2}\right)=c Y^{2}$, то очевидно, что $\omega_{1}\left(z_{i}\right)=c z_{i}$ при $i=1, \ldots, 4$. Согласно $[15]$ в $\mathfrak{g l}(\lambda)$ выполнены соотношения

$$
\begin{aligned}
3\left[z_{1}, z_{2}\right]-2\left[z, z_{3}\right] & =24\left(\lambda^{2}-4\right) Y, \\
4\left[z_{3},\left[z, z_{1}\right]\right]-3\left[z_{2},\left[z, z_{2}\right]\right] & =576\left(\lambda^{2}-9\right) z .
\end{aligned}
$$

Применив $\omega_{1}$ к обеим частям этих соотношений, получим

$$
\begin{aligned}
c^{2}\left(\lambda^{2}-4\right) & =\bar{\lambda}^{2}-4, \\
c^{3}\left(\lambda^{2}-9\right) & =c\left(\bar{\lambda}^{2}-9\right),
\end{aligned}
$$

откуда $c^{2}=1$ и $\lambda^{2}=\bar{\lambda}^{2}$. Теорема доказана.

СлеДСтвИЕ 6.1. Если $\lambda^{2} \neq \bar{\lambda}^{2}$, mо $\mathfrak{g l}(\lambda)$ не имеет инволютивных антилинейных автоморфизмов.

ДОКАЗАТЕЛЬСТВО. Пусть $\omega$ - инволютивный антилинейный автоморфизм; очевидно, что $\tau: X \leftrightarrow Y$ и $\tau(H)=H$ определяет антиавтоморфизм алгебры $\mathfrak{g l}(\lambda)$. Тогда $\tau \circ \omega-$ инволютивный антилинейный автоморфизм, что и требовалось доказать.

Если $\lambda^{2}=\bar{\lambda}^{2}$, то $\mathfrak{A}_{\lambda}$ обладает инволютивным антилинейным автоморфизм $\omega$ :

$$
\omega(X)=Y, \quad \omega(Y)=X, \quad \omega(H)=H .
$$

В дальнейшем мы будем рассматривать унитарные модули относительно такого автоморфизма.

TEOPEMA 6.3. Пусть $\lambda^{2} \in \mathbb{R}$ u nусть $F_{\theta}(t)=R(t) /\left(1-e^{-2 t}\right)$, где $R(t)-\kappa в а з и$ многочлен, $y$ которого ни одна из экспонент $s_{i}$ не принадлежит $\Lambda=[\lambda-1] \cap$ $[\lambda+1] \cap[-\lambda-1] \cap[-\lambda+1] . \quad \mathfrak{g l}(\lambda)-$ Модуль с характером $F_{\theta}(t)$ унитарен, если и только если

$$
F_{\theta}(t)=\sum n_{i} \frac{e^{-(\lambda+1) t}-e^{s_{i} t}}{1-e^{-2 t}}, \quad \text { де } \quad n_{i} \in \mathbb{Z}_{+} .
$$


ДокАЗАТЕЛЬСтво. Пусть $V$ - неприводимый модуль с характером $F_{\theta}(t)$, а $P(H)$ аннигилятор $Y v$, где $v$ - вектор старшего веса. Тогда $P(H)$ - характеристический многочлен оператора $H$ в $V_{-1}$. Однако поскольку унитарная форма инвариантна и $\omega(H)=H, H$ является самосопряженным; следовательно, все корни многочлена $P$ вешественны. Далее, если $\alpha$ - кратный корень кратности $m>1$, многочлен $P$ имеет вид $P=(H-\alpha)^{m} Q(H)$. При $u=(H-\alpha)^{m-1} Q(H) v$ имеем

$\langle u, u\rangle=\left\langle(H-\alpha)^{m-1} Q(H) v,(H-\alpha)^{m-1} Q(H) v\right\rangle=\left\langle Q(H) v,(H-\alpha)^{2 m-2} Q(H) v\right\rangle=0$.

Вследствие эрмитовости $u=0$. Таким образом, $m=1$.

Согласно теореме $5.2 V$ имеет вид $V\left(s_{1}\right) \otimes \cdots \otimes V\left(s_{k}\right)$, где $V\left(s_{i}\right)$ - неприводимые $\widehat{\mathfrak{g l}}(\infty)$-модули. Очевидно, что $V$ унитарен, если и только если каждый $V\left(s_{i}\right)$ унитарен. Далее, согласно работе [14] мы знаем, что $\widehat{\mathfrak{g l}}(\infty)$-модуль $V(s)$ унитарен, если и только если $\theta_{i}-\theta_{i+1}+\delta_{i 0} c \in \mathbb{Z}_{+}$при любых $i$, где $\theta_{i}-$ координаты старшего веса, а $c$ - значение центрального заряда. Теорема доказана.

\section{7. АЛГЕБРА $\mathfrak{g}(\lambda)$ И СИММЕТРИЧЕСКАЯ ГРУППА}

В данном разделе мы строим явную реализацию некоторых неприводимых $\mathfrak{g l}(\lambda)$-модулей. А именно мы раскладываем тензорные степени модуля Верма над $\mathfrak{s l}(2)$ и указываем соответствуюшие характеристические многочлены и $q$-характеры.

Лемма 7.1. Пусть $A$ - ассоциативная алгебра, пусть $\mathfrak{S}_{n}$ действует естественным образом на $A^{\otimes n}$. Тогда алгебра $\mathfrak{S}_{n}$-инвариантов $\left(A^{\otimes n}\right)^{\mathfrak{S}_{n}}$ порождается әлементами вида

$$
a \otimes 1 \otimes 1 \otimes \cdots \otimes 1+\cdots+1 \otimes 1 \otimes 1 \otimes \cdots \otimes a .
$$

ДОКАЗАТЕЛЬСТво. Обозначим

$$
s\left(a_{1}, \ldots, a_{n}\right)=\sum_{\sigma \in \mathfrak{S}_{n}} a_{\sigma(1)} \otimes \cdots \otimes a_{\sigma(n)} .
$$

Пусть $B$ - алгебра, порожденная элементами $s(a, 1, \ldots, 1)$ при любых $a \in A$. Пусть $\left|s\left(a_{1}, \ldots, a_{n}\right)\right|$ - число элементов $a_{i}$, отличных от 1 . Докажем, что $B \simeq\left(A^{\otimes n}\right)^{\mathfrak{S}_{n}}$.

Индукцией по $\left|s\left(a_{1}, \ldots, a_{n}\right)\right|$ покажем, что $s\left(a_{1}, \ldots, a_{n}\right) \in B$. Действительно, если $\left|s\left(a_{1}, \ldots, a_{n}\right)\right|=1$, то по определению $s\left(a_{1}, \ldots, a_{n}\right)=s(a, 1, \ldots, 1) \in B . \quad$ Пусть $\left|s\left(a_{1}, \ldots, a_{n}\right)\right|=l>1$. Рассмотрим

$$
s\left(a_{1}, \ldots, a_{l-1}, 1, \ldots, 1\right) s\left(a_{l}, 1, \ldots, 1\right)=\alpha s\left(a_{1}, \ldots, a_{l}, 1, \ldots, 1\right)+\cdots,
$$

где $\alpha$ - отличная от нуля постоянная, а точки означают линейную комбинацию членов $s\left(b_{1}, \ldots, b_{m}, 1, \ldots, 1\right)$, в которых $m<l$, что и требовалось доказать. 
Теорема 7.1. Пусть $V=M^{\lambda-1}$ - модуль Верма со стариим весом $\lambda-1$ над $\mathfrak{s l}(2)(u \mathfrak{g l}(\lambda))$. Тогда

$$
V^{\otimes n}=\bigoplus_{\nu} V^{\nu} \otimes S^{\nu}
$$

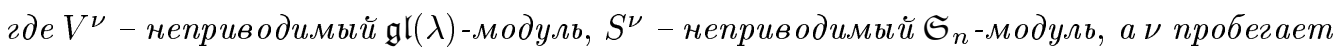
разбиения $n$.

ДокАЗАТЕЛЬСтво. Ясно, что $V$ неприводим не только как $\mathfrak{g l}(\lambda)$-модуль, но и как



Образ $U(\mathfrak{g l}(\lambda))$ в $\operatorname{End}(W)$ соответствует подалгебре, порожденной элементами $s(a, 1, \ldots, 1)$ при $a \in \mathfrak{g l}(\lambda)$, следовательно, согласно лемме 7.1 он изоморфен $\left(\mathfrak{A}_{\lambda}^{\otimes n}\right)^{\mathfrak{S}_{n}}$.

Разложим $W$ на изотопические $\mathfrak{S}_{n}$-модули $W=\bigoplus_{\nu} W^{\nu}$ и представим каждый $W^{\nu}$ в виде $W^{\nu}=V^{\nu} \otimes S^{\nu}$, где $V^{\nu}$ - неприводимый $\mathfrak{g l}(\lambda)$-модуль. Такое представление не является единственным; мы можем, например, положить $V^{\nu}=e_{\nu}(W)$ для всех минимальных идемпотентов в $\mathbb{C}\left[\mathfrak{S}_{n}\right]$, соответствуюших разбиению $\nu$.

Чтобы показать, что $V^{\nu}$ неприводим как $\mathfrak{g l}(\lambda)$-модуль, рассмотрим $V_{1}^{\nu}=$ $\operatorname{Hom}_{\mathfrak{S}_{n}}\left(S^{\nu}, W\right)$. Как $\mathfrak{g l}(\lambda)$-модуль $V_{1}^{\nu}$ изоморфен модулю $V^{\nu}$. Пусть $\phi, \psi \in V_{1}^{\nu}$ и $\phi \neq 0$. Покажем, что сушествует вектор $u \in U(\mathfrak{g l}(\lambda))$ такой, что $u \phi=\psi$. Действительно, поскольку $W$ неприводим как $\mathfrak{A}_{\lambda}^{\otimes n}$-модуль, теорема плотности $[16$, гл. XVII, $\S 3$, теорема 1$]$ утверждает, что существует $w \in \mathfrak{A}_{\lambda}^{\otimes n}$ такой, что $w \phi\left(v_{i}\right)=\psi\left(v_{i}\right)$, где $v_{i}$ образуют базис в $S^{\nu}$. (Поскольку $S^{\nu}$ неприводим и $\phi \neq 0$, векторы $\psi\left(v_{i}\right)$ являются линейно независимыми при $i=1, \ldots, \operatorname{dim} S^{\nu}$.)

Усредним элемент $w \phi$ по $\mathfrak{S}_{n}$ :

$$
(w \phi)^{\#}=\frac{1}{\left|\mathfrak{S}_{n}\right|} \sum \sigma(w \phi) \sigma^{-1}=\frac{1}{\left|\mathfrak{S}_{n}\right|} \sum \sigma(w) \sigma^{-1}(\phi)=w^{\#} \phi
$$

С другой стороны, поскольку $w \phi=\psi$, мы видим, что $(w \phi)^{\#}=(\psi)^{\#}=\psi$, т.е. $w^{\#} \phi=\psi$, и мы можем считать, что $w \in\left(\mathfrak{A}_{\lambda}^{\otimes n}\right)^{\mathfrak{S}_{n}}$. Таким образом, сушествует вектор $u \in U(\mathfrak{g l}(\lambda))$ такой, что $u \phi=\psi$. Другими словами, $V_{1}^{\nu}$ неприводим, что и требовалось доказать.

СлЕДСТВИЕ 7.1.

1. Пусть $\nu=\left(\nu_{1}, \ldots, \nu_{n}\right)$. Производящая функиия, соответствующая стариему весу $\nu$, имеет вид

$$
\sum_{i=1}^{n} \nu_{i} e^{(\lambda-2 i+1) t}
$$

2. Представим $\nu$ в виде $\nu=\left(\theta_{1}^{\alpha_{1}} \ldots \theta_{m}^{\alpha_{m}}\right)$, где $\theta_{1}>\cdots>\theta_{m}>0$ и $\alpha_{i} \neq 0$ для любых $i$, а $\theta^{\alpha}$ обозначает произведение $\theta \ldots \theta$ из $\alpha$ әлементов. Тогда характеристический многочлен модуля $V^{\nu}$ есть

$$
P(H)=\prod_{i=1}^{m}\left(H-\lambda-2 \alpha_{1}-2 \alpha_{2}-\ldots-2 \alpha_{i}-1\right) .
$$


ДокАЗАтЕльство. Утверждение 1 следует из теоремы 4.3. Для доказательства утверждения 2 умножим (7.1) на $1-e^{-2 t}$ и получим

$$
\nu_{1} e^{(\lambda-1) t}+\sum_{i=1}^{n-1}\left(\nu_{i+1}-\nu_{i}\right) e^{(\lambda-2 i+1) t}-\nu_{n} e^{(\lambda-2 n-1) t} .
$$

Следовательно сумма (7.2) без первого слагаемого является решением обыкновенного дифференциального уравнения с постоянными коэффициентами, чье характеристическое уравнение имеет в точности указанный вид. Следствие 7.1 доказано.

СЛЕДСТВИЕ 7.2. Пусть $a=e^{\lambda} u q=e^{-\alpha}$, әде $\alpha$ - положительньй корень алгебpbl $\mathfrak{s l}(2)$. Тогда q-характер модуля $V^{\nu}$ есть

$$
\chi_{\nu}=\frac{a^{|\nu|} q^{n(\nu)}}{\prod_{x \in \nu}\left(1-q^{h(x)}\right)},
$$

где $|\nu|$ - число клеток в диаграмме Юнга, соответствующей $\nu$,

$$
n(\nu)=\sum_{i \geqslant 1} \nu_{i}
$$

a $h(x)$ - длина крюка, соответствующего клетке $x$.

ДокАЗАТЕЛЬСтво. Согласно работе [17, пример 2 в разд. 3$]$ для $S$-функции $s_{\nu}$ имеем

$$
\chi_{\nu}=s_{\nu}\left(a, a q, a q^{2}, \ldots\right)=a^{|\nu|} s_{\nu}\left(1, q, q^{2}, \ldots\right),
$$

что и равно правой части формулы (7.3). Следствие доказано.

\section{8. ОРТОГОНАЛЬНЫЕ МНОГОЧЛЕНЫ ДЛЯ АЛГЕБРЫ $\mathfrak{g l}(n)$}

ТЕОРЕМА 8.1. В алгебре $\mathfrak{g l}(n)$ имеют место следующие утвержсдения:

I. Для базиса $e_{k l}=(\operatorname{ad} Y)^{k-l}\left(X^{k}\right)$, где $0 \leqslant k \leqslant n-1 u-k \leqslant l \leqslant k$, имеем

$$
\left\langle e_{k l}, e_{k^{\prime} l^{\prime}}\right\rangle=\delta_{k, k^{\prime}} \delta_{l+l^{\prime}, 0} .
$$

II. Пусть әлементы $f_{k l}$ определяются уравнениями $(0 \leqslant l \leqslant k)$

$$
\begin{aligned}
& (\operatorname{ad} Y)^{k-l}\left(X^{k}\right)=X^{l} f_{k l}, \\
& (\operatorname{ad} Y)^{k+l}\left(X^{k}\right)=f_{k,-l} Y^{l} .
\end{aligned}
$$

Положим $T_{i}(H)=\left(n^{2}-(H+2 i-1)^{2}\right) / 4 u \alpha_{i}=n-2 i+1$ при $i=1, \ldots, n$. При фиксированном $l \geqslant 0$ илюбых $k \geqslant l$ многочлены $f_{k l}$ образуют базис, ортогональный относительно формы

$$
\langle f, g\rangle= \begin{cases}\sum_{i=l+1}^{n} f\left(\alpha_{i}\right) g\left(\alpha_{i}\right) T_{1}\left(\alpha_{i}\right) \ldots T_{l}\left(\alpha_{i}\right) & \text { для } l>0 \\ \sum_{i=1}^{n} f\left(\alpha_{i}\right) g\left(\alpha_{i}\right) & \text { для } \quad l=0 .\end{cases}
$$


III. С точностью до постоянного множителя многочлены $f_{k l}$ соответствуют многочленам Хана одной дискретной переменной

$$
f_{k l}(H)={ }_{3} F_{2}\left(\begin{array}{c|c}
l-k, l+k+1, \frac{1}{2}(1-n-H) & 1 \\
l+1, l+1-n & 1
\end{array}\right) \times T_{0}\left(\alpha_{l+1}\right) \ldots T_{0}\left(\alpha_{k}\right),
$$

$2 \partial e$

$$
{ }_{3} F_{2}\left(\begin{array}{c}
\alpha_{1}, \alpha_{2}, \alpha_{3} \\
\beta_{1}, \beta_{2}
\end{array} \mid z\right)=\sum_{i=0}^{\infty} \frac{\left(\alpha_{1}\right)_{i}\left(\alpha_{2}\right)_{i}\left(\alpha_{3}\right)_{i}}{\left(\beta_{1}\right)_{i}\left(\beta_{2}\right)_{i}} \frac{z^{i}}{i !}
$$

- обобщенная гипергеометрическая функиия, $(\alpha)_{0}=1 u(\alpha)_{i}=\alpha(\alpha+1) \ldots(\alpha+i-1)$ при $i>0$.

ДокАЗАТЕльство. І. Заметим, во-первых, что подпространства $L^{2 k}$ в разложении $\mathfrak{g l}(n)=L^{0} \oplus L^{2} \oplus \cdots \oplus L^{2 n-2}$ попарно ортогональны. Действительно, форма $A, B \mapsto$ $\operatorname{tr} A B$ определяет инвариантное спаривание пространств $L^{k}$ и $L^{l}$ и, следовательно, $\mathfrak{s l}(2)$ гомоморфизм $L^{k} \rightarrow L^{l}$, что возможно только при $k=l$. Более того, ясно, что $\left\langle\mathfrak{g l}(n)_{k}, \mathfrak{g l}(n)_{l}\right\rangle \neq 0$, если и только если $k+l=0$. Теперь заметим, что $\mathfrak{g l}(n)_{l} \cap L^{2 k}=$ $\operatorname{Span}\left((\operatorname{ad} Y)^{k-l}(X)\right)$. Это доказьвает утверждение I.

II. Пусть $X^{l} f(H) \in \mathfrak{g l}(n)_{l}$ и $g(H) Y^{l} \in \mathfrak{g l}(n)_{-l}$. Тогда

$$
\operatorname{tr}\left(g(H) Y^{l} X^{l} f(H)\right)=\operatorname{tr}\left(f(H) g(H) T_{1}(H) \ldots T_{l}(H)\right) .
$$

Как легко проверить,

$$
\operatorname{tr} f(H)=\sum_{1 \leqslant i \leqslant n} f\left(\alpha_{i}\right) \quad \text { для любых } f(H) \in \mathfrak{g l}(n)_{0} .
$$

Отсюда следует (8.1).

Более того, утверждение I означает, что для любого фиксированного $l$, лежащего между $-k$ и $k$, многочлены $f_{k l}$ и $f_{k,-l}$ образуют два двойственных базиса, т.е. $\left\langle f_{k l}, f_{k l^{\prime}}\right\rangle=\delta_{l+l^{\prime}, 0}$. Однако степени многочленов $f_{k l}$ и $f_{k,-l}$ равны, что означает совпадение этих многочленов с точностью до множителя. Это доказьвает утверждение II.

Утверждение III следует из того факта, что ортогональные многочлены однозначно определяются весовой функцией и интервалом, на котором рассматривается скалярное произведение. Уравнение (8.2) следует из сравнения коэффициентов при старших членах. Теорема 8.1 доказана.

ЗАмЕчАнИЕ. Многочлены Хана определены для трех параметров $\alpha, \beta$ и $N$ следующим образом:

$$
h_{p}^{(\alpha, \beta)}(z, N)=\frac{(-1)^{p} \Gamma(N)(\beta)_{p}}{p ! \Gamma(N-p)}{ }_{3} F_{2}\left(\begin{array}{c}
-p, \alpha+\beta+p+1,-z \\
\beta+1, l-N
\end{array} \mid 1\right) .
$$

Наши многочлены $f_{k l}$ совпадают с точностью до множителя с $h_{p}^{(\alpha, \beta)}(z, N)$ при $\alpha=$ $\beta=l, p=k-l, z=(H+n-1) / 2$ и $N=n-1$.

Теперь покажем, как основные свойства многочленов Хана следуют из теории представлений алгебры $\mathfrak{s l}(2)$. Для любого $f(H) \in \mathbb{C}[H]$ положим

$$
\Delta f(H)=f(H+2)-f(H), \quad \nabla f(H)=f(H)-f(H-2) .
$$


Tеорема 8.2. Рассмотрим $\mathfrak{g l}(n)$ как $\mathfrak{s l}(2)$-модуль относительно образа главно-



I. Оператор $\Omega$ является самосопряженныц по отночению $к$ форме $\langle\cdot, \cdot\rangle$, а многочлены $X^{l} f_{k l}$ - собственные функции $\Omega$, соответствующие собственным значениям $2 k(k+1)$. Многочлены $f_{k l}$ удовлетворяют разностному уравнению

$$
T_{0}(H) \nabla \Delta(f)-(l+1)(H+l) \Delta(f)+(k-l)(k+l+1) f=0 .
$$

II. Имеет место формула

$$
f_{k l}=\left\{\begin{array}{lll}
\frac{\nabla^{k-l}\left(T_{1} \ldots T_{k}\right)}{T_{1} \ldots T_{l}}, & \text { ecлu } & l>0, \\
\nabla^{k}\left(T_{1} \ldots T_{k}\right), & \text { если } & l=0 .
\end{array}\right.
$$

III. Имеет место формула

$$
\left\langle f_{k l}, f_{k l}\right\rangle=\frac{(k-l) !(k !)^{2}}{(k+l) !(2 k+1)} n\left(n^{2}-1^{2}\right) \ldots\left(n^{2}-k^{2}\right) .
$$

ДокаЗАтельство. I. Поскольку форма $\langle\cdot, \cdot\rangle \quad \mathfrak{s l}(2)$-инвариантна, $\langle[w, u], v\rangle=$ $-\langle u,[w, v]\rangle$ при любых $u, v \in \mathfrak{g l}(n)$ и $w \in \mathfrak{s l}(2)$. Через $*$ обозначим $U(\mathfrak{s l}(2))$-действие на $\mathfrak{g l}(n) ;$ по индукции заключаем, что $\langle w * u, v\rangle=-\left\langle u, w^{t} * v\right\rangle$, где теперь $w \in U(\mathfrak{s l}(2))$, a $t$ - главная антиинволюция. Поскольку, как легко проверить, $\Omega^{t}=\Omega$, мы имеем $\langle\Omega * u, v\rangle=\langle u, \Omega * v\rangle$, т.е. оператор $\Omega$ является самосопряженным. Так как $\mathfrak{g l}(n)=\oplus L^{2 i}$ и $X^{l} f_{k l}=(\operatorname{ad} Y)^{k-l}\left(X^{k}\right) \in L^{2 k}$, мы видим, что $X^{l} f_{k l}$ является собственной функцией оператора $\Omega$ с собственным значением $2 k(k+1)$. Применяя $\Omega$ к $X^{l} f_{k l}$, получаем уравнение (8.6).

II. Напомним тождество

$$
(\operatorname{ad} y)^{p}(a)=\sum_{j=0}^{p}(-1)^{j}\left(\begin{array}{c}
p \\
j
\end{array}\right) y^{p-j} a y^{j} .
$$

Положим в нем $a=X^{k}, p=k-l$ и умножим на $Y^{l}$ слева. Получим

$$
\begin{aligned}
Y^{l} X^{l} f_{k l} & =Y^{l}(\operatorname{ad} Y)^{k-l}\left(X^{k}\right)=Y^{l}\left(\sum_{j=0}^{k-l}(-1)^{j}\left(\begin{array}{c}
k-l \\
j
\end{array}\right) Y^{k-l-j} X^{k} Y^{j}\right)= \\
& =\sum_{j=0}^{k-l}(-1)^{j}\left(\begin{array}{c}
k-l \\
j
\end{array}\right) y^{k-j} X^{k} Y^{j}=\sum_{j=0}^{k}(-1)^{j}\left(\begin{array}{c}
k \\
j
\end{array}\right) T_{j-1} \ldots T_{0} T_{1} \ldots T_{k-j} .
\end{aligned}
$$

Однако

$$
\nabla^{k-l}(f)(H)=\sum_{j=0}^{k}(-1)^{j}\left(\begin{array}{l}
k \\
j
\end{array}\right) f(H-2 j)
$$

для любой функции $f$ и, следовательно,

$$
Y^{l} X^{l} f_{k l}=\nabla^{k-l}\left(T_{1} \ldots T_{k}\right) .
$$


Поскольку $Y^{l} X^{l}=T_{1} \ldots T_{l}$, утверждение II доказано.

III. Модуль $L^{2 k}$ является линейной оболочкой векторов $v_{l}=(\operatorname{ad} Y)^{k-l}\left(X^{k}\right)$ при $-k \leqslant$ $l \leqslant k ;$ следовательно,

$$
\left\langle v_{l}, v_{m}\right\rangle=-\left\langle v_{l-1}, v_{m+1}\right\rangle=\cdots=(-1)^{l}\left\langle v_{0}, v_{l+m}\right\rangle
$$

согласно инвариантности формы. Таким образом, $\left\langle v_{l}, v_{-l}\right\rangle=(-1)^{l}\left\langle v_{0}, v_{0}\right\rangle$. Однако $v_{l}=$ $X^{l} f_{k l}=(\operatorname{ad} Y)^{k-l}\left(X^{k}\right)$ и $v_{-l}=f_{k,-l} Y^{l}=(\operatorname{ad} Y)^{k+l}\left(X^{k}\right)$.

Теперь напомним, что многочлены $f_{k l}$ и $f_{k,-l}$ совпадают с точностью до постоянного множителя, и сравним старшие коэффициенты. Мы видим, что

$$
f_{k,-l}=(-1)^{l} \frac{(k+l) !}{(k-l) !} f_{k, l} .
$$

Следовательно,

$$
\left\langle f_{k, l}, f_{k, l}\right\rangle=\operatorname{tr}\left(f_{k, l} Y^{l} \cdot X^{l} f_{k, l}\right)=(-1)^{l} \frac{(k+l) !}{(k-l) !}\left\langle v_{l}, v_{-l}\right\rangle=\frac{(k+l) !}{(k-l) !}\left\langle v_{0}, v_{0}\right\rangle .
$$

Остается показать, что

$$
\left\langle v_{0}, v_{0}\right\rangle=\frac{n\left(n^{2}-1^{2}\right) \ldots\left(n^{2}-k^{2}\right)}{2 k+1}(k !)^{2} .
$$

Очевидно, что $f_{k}=\left((\operatorname{ad} Y)^{k}\left(X^{k}\right)\right)^{2} \in U(\mathfrak{s l}(2))$, отсюда $\left\langle v_{0}, v_{0}\right\rangle=\operatorname{tr}_{n} \phi_{n}\left(f_{k}\right)$, где $\operatorname{tr}_{n}-$ след на $\mathfrak{g l}(n)$ и $\phi_{n}$ - гомоморфизм из $U(\mathfrak{s l}(2))$ в $\mathfrak{g l}(n)$, индуцированный главным вложением. Теперь ясно, что $P_{k}(n)=\operatorname{tr}_{n}\left(\phi_{n}\left(f_{k}\right)\right)$ - многочлен степени $2 k+1$. Более того, этот многочлен является нечетным. Однако $\phi_{n}\left(X^{k}\right)=0$ при $n \leqslant k$ и $\phi_{n}\left(f_{k}\right)=0$. Следовательно, $P_{k}(n)=0$, если $n \leqslant k$, и $P_{k}(-n)=0$, потому что $P_{k}$-нечетный многочлен. Отсюда имеем

$$
P_{k}(n)=c_{k} n\left(n^{2}-1^{2}\right) \ldots\left(n^{2}-k^{2}\right) .
$$

Чтобы найти постоянную $c_{k}$, достаточно вычислить $P_{k}(k+1)=\operatorname{tr}_{k+1}\left(\phi_{k+1}\left(f_{k}\right)\right)$. Однако в этом случае $X^{k}=(k !)^{2} e_{1, k+1}$ и

$$
(\operatorname{ad} Y)^{k}\left(X^{k}\right)=(k !)^{2}(\operatorname{ad} Y)^{k}\left(e_{1, k+1}\right)=(k !)^{2} \sum_{j=0}^{k}(-1)^{j}\left(\begin{array}{l}
k \\
j
\end{array}\right) e_{k+1-j, k+1-j} .
$$

Таким образом,

$$
\operatorname{tr}_{k+1}\left(\phi_{k+1}\left(f_{k}\right)\right)=(k !)^{4} \sum_{j=0}^{k}\left(\begin{array}{c}
k \\
j
\end{array}\right)^{2}=(k !)^{4}\left(\begin{array}{c}
2 k \\
k
\end{array}\right),
$$

откуда следует, что

$$
c_{k}=\frac{(k !)^{2}}{2 k+1} .
$$

Теорема 8.2 доказана. 
ПРЕДЛОЖЕНИЕ 8.1. Имеют место соотношения ортогональности:

$$
\sum_{i} f_{k l}\left(\alpha_{i}\right) f_{k_{1}, l}\left(\alpha_{i}\right) T_{1}\left(\alpha_{i}\right) \ldots T_{l}\left(\alpha_{i}\right)=\delta_{k, k_{1}} c_{k, l}
$$

əде $c_{k, l}=\frac{(k-l) !(k !)^{2}}{(k+l) !(2 k+1)} n\left(n^{2}-1^{2}\right) \ldots\left(n^{2}-k^{2}\right)$;

$$
\sum_{k=l}^{n-1} \frac{1}{c_{k l}} f_{k l}\left(\alpha_{i}\right) f_{k l}\left(\alpha_{j}\right) T_{1}\left(\alpha_{i}\right) \ldots T_{l}\left(\alpha_{i}\right)=\delta_{i j} \quad \text { для любъц } \quad i, j>l .
$$

ДокАЗАТЕльство. Формула (8.8) следует из теоремы 8.2 (утверждение III). Чтобы доказать формулу (8.9), выразим $e_{i j}$ через $X^{l} f_{k l}$ и $f_{k,-l} Y^{l}$. Имеем, в частности,

$$
e_{i, i+l}=\sum_{k=l}^{n-1} \alpha_{i k} X^{l} f_{k l}
$$

для любых $k$ между $l$ и $n-1$. Однако, как легко проверить,

$$
\begin{aligned}
f_{k l} Y^{l} & =\left(\sum_{i=1}^{n} f_{k l}\left(\alpha_{i}\right) e_{i i}\right)\left(\sum_{i=1}^{n-1} e_{i+1, i}\right)^{l}= \\
& =\left(\sum_{i=1}^{n} f_{k l}\left(\alpha_{i}\right) e_{i i}\right)\left(\sum_{i=1}^{n-l} e_{i+l, i}\right)=\sum_{i=1}^{n-l} f_{k l}\left(\alpha_{i+l}\right) e_{i+l, i} .
\end{aligned}
$$

Следовательно, согласно теореме 8.2 имеем

$$
f_{k l}\left(\alpha_{i+l}\right)=\left\langle e_{i, i+l}, f_{k l} Y^{l}\right\rangle=\alpha_{i k}\left\langle f_{k l} Y^{l}, X^{l} f_{k l}\right\rangle=\alpha_{i k} c_{k l} .
$$

Другими словами,

$$
e_{i, i+l}=\sum_{k=l}^{n-1} \frac{f_{k l}\left(\alpha_{i+l}\right)}{c_{k l}} X^{l} f_{k l}
$$

Аналогично можно записать

$$
e_{i+l, i}=\sum_{k=l}^{n-1} \frac{f_{k l}\left(\alpha_{i+l}\right) T_{1}\left(\alpha_{i+1}\right) \ldots T_{l}\left(\alpha_{i+l-1}\right)}{c_{k l}} f_{k l} Y^{l}
$$

Поэтому, полагая $i^{\prime}=i+l, j^{\prime}=j+l$, заключаем, что

$$
\begin{aligned}
\left\langle e_{i+l, i}, e_{j, j+l}\right\rangle & =\sum_{i=l}^{n-1} \frac{1}{c_{k l}} f_{k l}\left(\alpha_{i+l}\right) f_{k l}\left(\alpha_{j+l}\right) T_{1}\left(\alpha_{i+1}\right) \ldots T_{l}\left(\alpha_{i+l-1}\right)= \\
& =\sum_{i=l}^{n-1} \frac{1}{c_{k l}} f_{k l}\left(\alpha_{i^{\prime}}\right) f_{k l}\left(\alpha_{j^{\prime}}\right) T_{1}\left(\alpha_{i^{\prime}-1}\right) \ldots T_{l}\left(\alpha_{i^{\prime}-l+1}\right)= \\
& =\sum_{i=l}^{n-1} \frac{1}{c_{k l}} f_{k l}\left(\alpha_{i^{\prime}}\right) f_{k l}\left(\alpha_{j^{\prime}}\right) T_{1}\left(\alpha_{i^{\prime}}\right) \ldots T_{l}\left(\alpha_{i^{\prime}}\right),
\end{aligned}
$$

что и требовалось доказать.

Выражая элементы Казимира $\Omega_{2}, \Omega_{3}, \ldots, \Omega_{n}$ алгебры $\mathfrak{g l}(n)$ через ортогональные многочлены, а не через матричные единицы, мы можем вывести различные тождества, связывающие эти многочлены. Некотрые из этих тождеств могут даже оказаться новыми, по крайней мере для нецелых значений $n$ (см. предположение 9.1 ниже). Например, для $\Omega_{2}$ имеет место следующее предложение. 
ПРЕДЛОЖЕНИЕ 8.2. В алгебре $\mathfrak{g l}(n)$ имеет место соотноиение

$$
\sum_{k=0}^{n-1}\left(\frac{1}{c_{k 0}} f_{k 0}^{2}+2 \sum_{l=1}^{k} \frac{1}{c_{k l}} f_{k l}^{2} T_{1} \ldots T_{l}-\frac{2 k+1}{n}\right)=-H .
$$

ДокАЗАТЕЛьство. Перепишем оператор Казимира для алгебры $\mathfrak{g l}(n)$, выраженный в терминах матричных элементов,

$$
\begin{aligned}
\Omega_{2} & =\sum_{k=1}^{n} e_{k k} \otimes e_{k k}+\sum_{i<j}\left(e_{i j} \otimes e_{j i}+e_{j i} \otimes e_{i j}\right)= \\
& =\sum_{k=1}^{n} e_{k k} \otimes e_{k k}+\sum_{k=1}^{n}(n-2 k+1) e_{k k}+2 \sum_{i<j} e_{j i} \otimes e_{i j}
\end{aligned}
$$

через ортогональные многочлены:

$$
\begin{aligned}
\Omega_{2} & =\sum_{k=0}^{n-1} \frac{1}{c_{k 0}} f_{k 0} \otimes f_{k 0}+\sum_{l=1}^{n-1} \sum_{k=l}^{n-1} \frac{1}{c_{k l}}\left(X^{l} f_{k l} \otimes f_{k l} Y^{l}+f_{k l} Y^{l} \otimes X^{l} f_{k l}\right)= \\
& =\sum_{k=0}^{n-1} \frac{1}{c_{k 0}} f_{k 0} \otimes f_{k 0}+2 \sum_{l=1}^{n-1} \sum_{k=l}^{n-1} \frac{1}{c_{k l}} f_{k l} Y^{l} \otimes X^{l} f_{k l}+\sum_{k>l} \frac{1}{c_{k l}}\left[X^{l} f_{k l}, f_{k l} Y^{l}\right] .
\end{aligned}
$$

Теперь преобразуем последнюю сумму. Для этого рассмотрим гомоморфизм $\varphi$ : $U(\mathfrak{g l}(n)) \rightarrow \mathfrak{g l}(n)$, индуцированный тождественным представлением. Поскольку

$$
\frac{1}{c_{k 0}} f_{k 0} \otimes f_{k 0}+\sum_{l=1}^{k} \frac{1}{c_{k l}}\left(X^{l} f_{k l} \otimes f_{k l} Y^{l}+f_{k l} Y^{l} \otimes X^{l} f_{k l}\right)
$$

представляет собой $\mathfrak{s l}(2)$-инвариант, его образ в $\mathfrak{g l}(n)$ также является $\mathfrak{s l}(2)$-инвариантом, т.е. постоянной величиной $D(k)$ :

$$
\frac{1}{c_{k 0}} f_{k 0} \cdot f_{k 0}+2 \sum_{l=1}^{k} \frac{1}{c_{k l}} f_{k l} Y^{l} \cdot X^{l} f_{k l}+\sum_{l=1}^{k} \frac{1}{c_{k l}}\left[X^{l} f_{k l}, f_{k l} Y^{l}\right]=D(k) .
$$

Вычисляя следы обеих частей, получаем

$$
n D(k)=\frac{1}{c_{k 0}} \operatorname{tr}\left(f_{k 0}^{2}\right)+2 \sum_{l=1}^{k} \frac{1}{c_{k l}} \operatorname{tr}\left(f_{k l}^{2} T_{1} \ldots T_{l}\right)=2 k+1,
$$

откуда

$$
D(k)=\frac{2 k+1}{n} .
$$

Поэтому

$$
\sum_{l=1}^{k} \frac{1}{c_{k l}}\left[X^{l} f_{k l}, f_{k l} Y^{l}\right]=\frac{2 k+1}{n}-\left(\frac{1}{c_{k 0}} f_{k 0} \cdot f_{k 0}+2 \sum_{l=1}^{k} \frac{1}{c_{k l}} f_{k l} Y^{l} \cdot X^{l} f_{k l}\right)
$$


Суммируя по $k$, получаем

$$
\sum_{k=0}^{n-1}\left(\frac{1}{c_{k 0}} f_{k 0} \cdot f_{k 0}+2 \sum_{l=1}^{k} \frac{1}{c_{k l}} f_{k l} Y^{l} \cdot X^{l} f_{k l}-\frac{2 k+1}{n}\right)=-\sum_{k=0}^{n-1} \sum_{l=1}^{k} \frac{1}{c_{k l}}\left[X^{l} f_{k l}, f_{k l} Y^{l}\right] .
$$

Линейные части одного и того же оператора Казимира, выраженные в различных базисах, совпадают, и, следовательно, последняя сумма равна

$$
-\sum_{i=1}^{n}(n-2 i+1) e_{i i}=-H
$$

что и требовалось доказать.

\section{9. ОРТОГОНАЛЬНЫЕ МНОГОЧЛЕНЫ И АЛГЕБРА $\mathfrak{g l}(\lambda)$}

Полученные выше результаты для $\mathfrak{g l}(n), n=1,2, \ldots$, имеют место с необходимыми изменениями и для $\mathfrak{g l}(\lambda)$ при любых комплексных $\lambda \notin \mathbb{Z} \backslash\{0\}$. В этом разделе будем рассматривать только такие значения $\lambda$.

Заметим, что алгебра $\mathfrak{g l}(\lambda)$ в отличие от алгебр $\mathfrak{g l}(\infty)$ или $\mathfrak{g l}^{ \pm}(\infty)$ не имеет базиса, состоящего из матричных единиц. Однако мы можем представить эту алгебру в виде

$$
\mathfrak{g l}(\lambda)=\bigoplus_{k=0}^{\infty} L^{2 k} \quad \text { и } \quad \mathfrak{g l}(\lambda)=\bigoplus_{i=-\infty}^{\infty} \mathfrak{g l}(\lambda)_{i}
$$

где $\mathfrak{g l}(\lambda)_{0}=\mathbb{C}[H]$, в то время как $\mathfrak{g l}(\lambda)_{i}=X^{i} \mathfrak{g l}(\lambda)_{0}$ и $\mathfrak{g l}(\lambda)_{-i}=\mathfrak{g l}(\lambda)_{0} Y^{i}$ при $i>0$.

Как было показано выше, сушествует след на алгебре $\mathfrak{g l}(\lambda)$, ограничение которого на $\mathfrak{g l}(\lambda)_{0}$ имеет производящую функцию

$$
\frac{e^{\lambda t}-e^{-\lambda t}}{e^{t}-e^{-t}}, \quad \text { если } \lambda \neq 0, \quad \text { и } \quad \frac{2 t}{e^{t}-e^{-t}}, \text { если } \lambda=0 .
$$

В первом случае мы нормировали след так, что $\operatorname{tr}(1)=\lambda$ по аналогии со случаем конечной размерности, когда скаляры естественным образом представлены скалярными матрицами и $\operatorname{tr}\left(1_{n}\right)=n$. Во втором случае, $\lambda=0$, мы считаем, что $\operatorname{tr}(1)=1$.

Заметим, что при цельх $\lambda=n$

$$
\frac{e^{n t}-e^{-n t}}{e^{t}-e^{-t}}=e^{(1-n) t}+e^{(3-n) t}+\cdots+e^{(n-3) t}+e^{(n-1) t}
$$

и соответствуюший функционал имеет вид

$$
\operatorname{tr}(f(H))=\sum_{i=1}^{n} f\left(\alpha_{i}\right), \quad \alpha_{i}=n-2 i+1 .
$$

Функционал $\operatorname{tr}$ на $\mathfrak{g l}(\lambda)$ порождает невырожденую симметричную инвариантую билинейную форму $\langle u, v\rangle=\operatorname{tr} u v$.

Теорема 9.1. В обозначениях раздела 8 имеем:

I. $\left\langle e_{k, l}, e_{k^{\prime}, l^{\prime}}\right\rangle=\delta_{k, k^{\prime}} \delta_{l+l^{\prime}, 0}$. 
II. Многочлены $f_{k, l}$ при фиксированном $l \geqslant 0 u k \geqslant l$ образуют ортогональный базис относительно скалярного произведения (8.1).

III. Многочлены $f_{k, l}$ совпадают с точностью до постоянного множителя $c$ непрерывными многочленами Хана дискретной переменной, которые определяются уравнениями (8.2) при $n=\lambda$.

ДокАЗАТЕЛЬСтво. Доказательство утверждений I и II аналогично доказательству соответствующих утверждений теоремы 8.1 при целых $\lambda$. Для доказательства утверждения III заметим, что для неотрицательного целого уравнение (8.2) выполняется согласно теореме 8.1. Однако обе части уравнения (8.2) являются многочленами от $H$, причем их коэффициенты - многочлены по $\lambda$. Вследствие непрерывности этих выражений в топологии Зариского наше утверждение доказано.

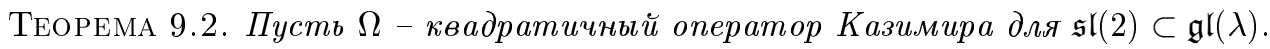

I. $\Omega$ является самосопряжсенным относительно формы (8.1), и многочлены $X^{l} f_{k l}$ nри $k \in \mathbb{Z}_{+} u l \in[-k, k] \cap \mathbb{Z}-$ собственные функиии $\Omega$ с собственными значениями $2 k(k+1)$. Многочлены $f_{k l}$ удовлетворяют разностному уравнению $(8.6)$ и имеют вид (8.7).

II. Имеет место формула

$$
\left\langle f_{k l}, f_{k l}\right\rangle=\left\{\begin{array}{lll}
\frac{(k-l) !}{(k+l) !} \frac{(k !)^{2}}{2 k+1} \lambda\left(\lambda^{2}-1^{2}\right) \ldots\left(\lambda^{2}-k^{2}\right), & \text { если } & \lambda \neq 0, \\
(-1)^{k} \frac{(k-l) !}{(k+l) !} \frac{(k !)^{4}}{2 k+1}, & \text { если } \quad \lambda=0 .
\end{array}\right.
$$

ДокАЗАТЕльство. Доказательство утверждения I аналогично доказательству соответствующих утверждений теоремы 8.2. Если $\lambda \neq 0$, то обе части уравнения (9.1) являются многочленами от $\lambda$, которые совпадают при целых $\lambda$ и, следовательно, равны по непрерывности в топологии Зариского. Чтобы охватить случай $\lambda=0$, рассмотрим $\lim _{\lambda \rightarrow 0}(\operatorname{tr} / \lambda)$; как и ранее, обе части уравнения становятся многочленами от $\lambda$, откуда следует утверждение II, что и требовалось доказать.

Несколько неожиданным является тот факт, что дуальные соотношения ортогональности выполнены при достаточно больших значениях $|\lambda|$. Действительно, положим

$$
c_{k l}= \begin{cases}\frac{(k-l) !}{(k+l) !} \frac{(k !)^{2}}{2 k+1}\left(\lambda^{2}-1^{2}\right) \ldots\left(\lambda^{2}-k^{2}\right), & \text { если } \lambda \neq 0, \\ (-1)^{k} \frac{(k-l) !}{(k+l) !} \frac{(k !)^{4}}{2 k+1}, & \text { если } \lambda=0 .\end{cases}
$$

ПРЕДПОЛОЖЕНИЕ 9.1. При достаточно больиих значениях $|\lambda|$ имеем $\left(\partial \Omega_{я} \alpha_{i}=\right.$ $\lambda-2 i+1)$

$$
\begin{gathered}
\sum_{k=l}^{\infty} \frac{1}{c_{k l}} f_{k l}\left(\alpha_{i}\right) f_{k l}\left(\alpha_{j}\right) T_{1}\left(\alpha_{i}\right) \ldots T_{l}\left(\alpha_{i}\right)=\delta_{i j} \quad \text { npu } \quad i, j>l, \\
\sum_{k=0}^{\infty}\left(\frac{1}{c_{k 0}} f_{k 0}\left(\alpha_{i}\right)^{2}+2 \sum_{l=1}^{k} \frac{1}{c_{k l}} f_{k l}\left(\alpha_{i}\right)^{2} T_{1}\left(\alpha_{i}\right) \ldots T_{l}\left(\alpha_{i}\right)-\frac{2 k+1}{\lambda}\right)=-\alpha_{i}
\end{gathered}
$$

nрu $i \in \mathbb{N}$. 
Априори $|\lambda|$ зависит от $l$. Нам не известно единообразного доказательства, мы проверили данное предположение только в некоторых частных случаях.

Из приведенных вьшше формул становится ясно, что если мы разделим форму $\langle\cdot, \cdot\rangle$ на $\lambda$ и рассмотрим многочлены только четной степени, то получим знакоопределенную форму не только при целых значениях $\lambda$, но и при таких вешественных значениях, при которых $0<|\lambda|<1$, а также при чисто мнимых $\lambda$. Последнее обстоятельство подсказывает деление следа на $\lambda$ с самого начала.

Благодарности. Мы благодарны NFR за финансовую поддержку, М. Васильеву за стимулируюший вопрос, П. Грозману и особенно В. Гердту (раздел 9), которые помогли нам с численными экспериментами с помощью программ Maple и MATHEMATICA. Представляется, что Марlе является программой, более подходяшей для наших задач.

\section{Список литературы}

[1] R. Roy. The work of Chebyshev on orthogonal polynomials. In: Topics in polynomials of one and several variables and their applications. Eds. Rassias Th. et. al. Singapore: World Scientific, 1993. Р. 495-512; Я. Л. Геронимус. Теория ортогональных многочленов. Обзор достижений отечественной математики. М.-Л.: Гостехтеориздат, 1950.

[2] А.Ф. Никифоров, С.К. Суслов, В.Б. Уваров. Классические ортогональные полиномы дискретной переменной. М.: Наука, 1985.

[3] B. Shoikhet. Certain topics on the Lie algebra $\mathfrak{g l}(\lambda)$ representation theory. q-alg/9703029.

[4] А.Ф.Никифоров, В. Б. Уваров. Специальные функции математической физики. М.: Наука, 1978.

[5] Б. Л. Фейгин. УМН. 1988. Т. 43. № 2. С. 157-158.

[6] J. Dixmier. J. Algebra. 1973. V. 24. P. 551-564.

[7] N. Ja. Vilenkin, A. U. Klimyk. Representation of Lie groups and special functions. Vol. 1-3. Dordrecht: Kluwer, 1991, 1992, 1993; Representation of Lie groups and special functions. Recent advances. Dordrecht: Kluwer, 1995.

[8] L. Littlejohn, A. Krall. Rocky Mt. J. Math. 1986. V. 16. № 3. P. 435-479; Acta Appl. Math. 1989. V. 17. P. 99-170.

[9] A. Mingarelli, A. Krall. Proc. Roy. Soc. Edinburgh. Sect. A. 1981. V. 90. P. 147-153.

[10] В. Деркач. Матем. заметки. 1998. Т. 64. № 4. С. 509-521.

[11] M. Vasiliev. Int. J. Mod. Phys. D. 1996. V. 5. P. 763-797.

[12] S. Montgomery. J. Algebra. 1997. V. 195. № 2. P. 558-579.

[13] J. Dixmier. Algèbres envellopentes. Paris: Gautier-Villars, 1974; Enveloping algebras. Providence, RI: AMS, 1996.

[14] V. Kač, A. Radul. Commun. Math. Phys. 1993. V. 157. P. 429-457.

[15] P. Grozman, D. Leites. Lie superalgebras of supermatrices of complex size and integrable dynamical systems. In: Complex Analysis and Related Topics (Proc. of the International Symposium, Cuernavaca, Mexica, November 18-22, 1996). Eds. N. Vasilevsky et. al. Basel: Birkhauser, 1999. P. $73-105$

[16] С. Ленг. Алгебра. М.: Мир, 1968.

[17] I. G. Macdonald. Symmetric functions and Hall polynomials. Second edition. With contributions by A. Zelevinsky. New York: Clarendon Press, Oxford University Press, 1995. 